\title{
Controlling the lifetime of antibubbles
}

\author{
Youen Vitry ${ }^{a}$, Stéphane Dorbolo ${ }^{b}$, Jan Vermant ${ }^{c}$ and Benoit Scheid $^{a}$ \\ ${ }^{a}$ TIPS C.P.165/67, Universit Libre de Bruxelles, Av. F. Roosevelt 50, 1050 Brussels, \\ Belgium \\ ${ }^{b}$ GRASP, Institute of Physics, Building B5a, Sart-Tilman, University of Liège, B4000 \\ Liège, Belgium \\ ${ }^{c}$ ETH Zürich, Dept. of materials, Vladimir-Prelog-Weg 5/10, 8093 Zürich, Switzerland
}

\begin{abstract}
An antibubble is a liquid droplet wrapped by a thin layer of gas, inside a bulk usually of the same liquid. The lifetime of an antibubble is governed by the drainage of the gas between the two liquid-gas interfaces populated by surfactants. Depending on the relative magnitude of surface viscosity and elasticity properties, which directly depend on or are determined by the nature of surfactants, the lifetime of an antibubble may vary a lot, from few seconds to few minutes. While experimental lifetimes are comparable whatever the dominating property, intrinsic differences exist as revealed by modeling. Nevertheless, these differences were not observed experimentally in previous studies, due to important sources of dispersion. In this review, the main sources of dispersion have been identified, such as (i) the initial amount of gas embedded in the antibubble, (ii) the level of saturation of gas in the bulk liquid, (iii) the presence of dust particles $(<0.5 \mu \mathrm{m})$ in the gas, and (iv) three-dimensional flow effects. By accounting for these various effects, the dependence of the antibubble radius on its lifetime is clarified together with the role of surface rheology. Results thus demonstrate that controlling the size and lifetime of antibubbles is achievable.
\end{abstract}

Keywords: Surfactants, Antibubbles, Drainage, Surface viscosity, Surface elasticity

\section{Contents}

\section{Introduction}


2 Theory 5

2.1 The model . . . . . . . . . . . . . . . 5

2.2 Parametric analysis . . . . . . . . . . . . . . . 8

2.3 Numerical results . . . . . . . . . . . . . . . . . 9

3 Experiments 14

3.1 Antibubble generator . . . . . . . . . . . . . . . . 14

3.2 Materials and Methods . . . . . . . . . . . . . . . . 16

3.3 Surfactant characterization . . . . . . . . . . . . . 17

3.4 Liquid viscosity . . . . . . . . . . . . . . . . . . . . . . . . 18

4 Results $\quad 19$

4.1 Influence of surface elasticity . . . . . . . . . . . . . . . 19

4.2 Influence of initial gas thickness . . . . . . . . . . . . 21

4.3 Influence of gas saturation . . . . . . . . . . . . . . 25

4.4 Influence of dust particles . . . . . . . . . . . . . . . . . . . . . . . . . 25

4.5 Other surfactants . . . . . . . . . . . . . . . . . . 29

4.6 Three-dimensional effects . . . . . . . . . . . . . . . . . . . 29

5 Conclusions $\quad 32$

\section{Appendix A}

Origin of the initial shell thickness in an antibubble

\section{Introduction}

The antibubble owns its name for being the opposite of a soap bubble. If a soap bubble is a thin spherical shell of soapy liquid surrounded by air, an antibubble is a thin spherical shell of air surrounded by liquid, as illustrated in Fig. 1. Antibubbles have first been reported in a scientific article by Hughes and Hughes in 1932 [1]. Even though antibubbles are extremely easy to make for an amateur scientist, as nicely reported in 1974 by Stong [2], not much evidences exist on the presence of antibubbles in nature. During the 80's in the context of boiling, Ida and Takashima [3] and Nosoko and Mori [4] formed antibubbles, without naming it, by dropping a denser volatile liquid onto a surface of another hot liquid. Noticeably, it has recently been suggested that leaks from subsea hydrocarbon production facilities contain antibubbles with specific acoustic signature [5]. Nowadays, benefiting from the development of microfluidics, several potential applications have arisen, 


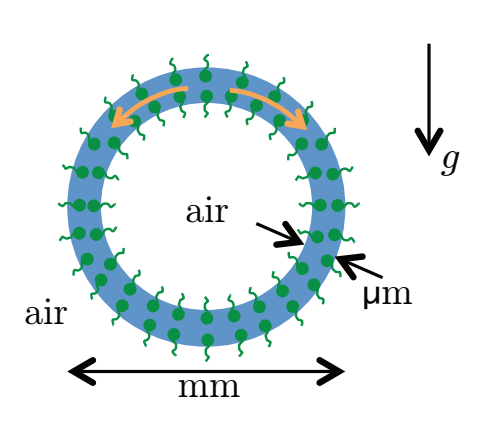

(a) Soap bubble

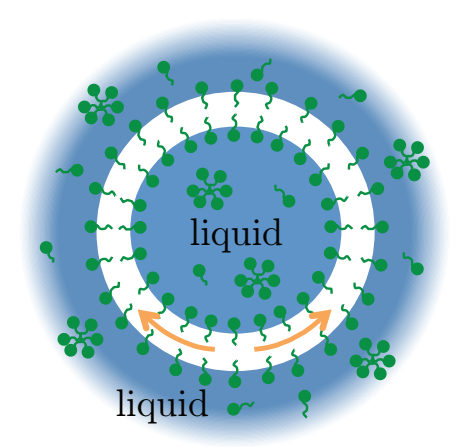

(b) Antibubble

Figure 1: Sketches of a soap bubble and an antibubble. Indicated typical dimensions in (a) are similar for (b). Surfactants are represented in green with their hydrophilic heads in the liquid and their hydrophobic tails in the air. The orange arrows indicate the direction of drainage under the action of gravity, $g$, in the liquid film (a) and in the air shell (b).

such as using antibubbles as contrast agent for enhancing ultrasonic imaging $[6,7]$ or loading the antibubble core with a ferrofluid to serve as a vehicle controlled by a magnetic field for drug delivery [8].

Several configurations have been proposed so far to produce antibubbles. The most classical one being a jet impacting a liquid surface and entraining a gas layer into a liquid bath, the antibubble resulting from the destabilization of the air-wrapped jet inside the liquid bath $[9,10]$. Alternatively, Tufaile and Sarterelli [11] described the formation of an antibubble as a result of a complex coalescence process between two rising bubbles formed from a submerged nozzle: as the second bubble coalesces with the first one, a spike of liquid penetrates into the first bubble, and destabilizes by inverted dripping into a droplet, forming the antibubble. Postema et al. [12] produced micronsize antibubbles by a similar mechanism of ultrasound-induced coalescence of microbubbles. Poortinga [13] and Silpe et al. [14] proposed another way to prepare micro-antibubbles by a two-step approach: a core-shell waterin-oil-in-water emulsion is first generated via microfluidics and freeze-dried thereafter to yield, upon subsequent reconstitution, an aqueous dispersion of antibubbles. Recently, Shen et al. [15] generated antibubbles at the outlet of a capillary dipped into a liquid and from which a Taylor flow is exiting: the antibubble is produced as a result of the breakup of the liquid column formed into the preceding bubble while still pinned at the tip of the capillary. Bai et al. [16] succeeded to produce antibubbles by shooting a liquid droplet onto a 
soap film, wrapping the drop with a shell of air: as the air-wrapped droplet reaches the liquid bath the external soap film coalesces with the surrounding liquid, forming the antibubble. Finally Beilharz et al. [17] determined the conditions to form antibubbles by impacting a highly viscous droplet into a liquid bath of low viscosity. Remarkably, they obtained antibubbles with pure liquids, i.e. without the use of surfactants. However, in such a case, the lifetime of antibubbles they observed was of the order of $100 \mathrm{~ms}$ only.

By choosing appropriate surface-active molecules, the lifetime of an antibubble can range from seconds to minutes [18], which is the time needed for the air to drain in its shell by the action of gravity from the bottom, called south pole, to the top, called north pole, where it accumulates, forming an air pocket [19]. An antibubble is therefore an ephemeral object, despite the attempt to keep it alive for hours, e.g., by shearing a confined antibubble in a spinning tube [2] or by Pickering stabilization of the interfaces with colloidal particles [20, 14]. Understanding what governs the lifetime of antibubbles with surfactants alone remains therefore of prior importance.

Investigations during the last decade and a half have started on the instabilities at birth and death of antibubbles [9] and on the statistics of their lifetimes [18], followed by an empirical description of factors affecting their stability [21], controlling their formation [10] or influencing their bouncing [22], and ended by experimental [23] and theoretical [24] investigations on their collapse. In most of the experimental studies however, commercial detergents have been used to stabilize antibubbles, making it difficult to draw any conclusions on the mechanism responsible for their long lifetime, as detergent properties and exact compositions are usually not well documented.

Consequently, it has not been fully clarified yet to what extent a single surfactant molecule is suitable for generating antibubbles and which of its properties is responsible for maintaining long lifetimes. In an attempt to answer this question, Dorbolo et al. [25] have demonstrated the crucial influence of surface (visco-elastic) modulus on the antibubble lifetime, having used a mixture of three components [26], among which myristic acid, which confers to the interface very high surface viscosity of the order of one mPa.s.m. Subsequently, the role of surface viscosity to the antibubble lifetime has been rationalized theoretically by Scheid et al. [27] who showed the good agreement with experimental data. Their model, based on the lubrication theory for the air film drainage, relies on two main assumptions: (i) the adsorption characteristic time is much smaller than the antibubble lifetime such as surface-tension gradients induced by drainage are neutralized by 
rapid replenishing of surfactants on the interface; (ii) the surface dilatational viscosity is of the same order of magnitude than the surface shear viscosity, this latter being the only one that could be measured with a surface rheometer. Even though the first hypothesis is compatible with the surfactant mixture used in [25] for which adsorption timescale has been shown to span between 0.1 and $1 \mathrm{~s}$ [28], it is not compatible with other surfactants like $\mathrm{C}_{12} \mathrm{E}_{6}$, with which antibubble lifetime is of the order of hundred seconds [18], i.e. comparable to their adsorption timescale [29]. Additionally, surface shear viscosity of small-molecule surfactants such as $\mathrm{C}_{12} \mathrm{E}_{6}$ is known to be extremely small, if not inviscid, as reported by Zell et al. [30]. Consequently, another effect than surface viscosity should be responsible for long lifetime of the order of minutes. This effect is the surface elasticity, triggered when surface tension gradients occur as surfactants are transported away from the south pole during the drainage, provoking Marangoni stresses that opposes in turn to the drainage.

The present paper investigates the role of surface elasticity in antibubble lifetime, in addition to other effects such as the influence of undersaturated liquid with air [31] and the influence of dust particles on the antibubble stability. Section 2 presents a general model as a combination of several particular models available in the literature, and shows numerical results demonstrating the crucial role of surface elasticity. Experimental settings are given in section 3, along with a new antibubble generator [32] and independent surface elasticity measurements. Results and comparisons with the model are presented in section 4 with sequential investigations of the influence of (i) surface elasticity, (ii) initial air film thickness, (iii) liquid saturation with gas, (iv) dust particle size, (v) other surfactant and (vi) three-dimensional effects, on the antibubble lifetime. Conclusions are drawn in section 5 .

\section{Theory}

\subsection{The model}

We use in this section the lubrication model developed in [27] that includes the effect of surface shear viscosity $\eta_{s}$, and extended in [31] to account for gas dissolution into the surrounding liquid. Using linear Boussinesq-Scriven surface fluid model [33], the model is further extended here to account for surface dilatational viscosity $\kappa_{s}$ and surface elasticity $E_{s}$. Because the antibubble radius $R$ remains constant during the antibubble lifetime, and the initial film thickness $h_{0}$ is at least three orders of magnitude smaller than 
the radius, the radial expansion of the interface is negligible, which actually results from the lubrication approximation at the leading order in the asymptotical expansion with respect to the aspect ratio $\varepsilon=h_{0} / R \ll 1$. Consequently, there is no contribution of the surface dilatational viscosity in the normal stress boundary condition [33], nor variation of surfactant concentration induced by radial expansion in the surfactant transport equation at the interface [34], as zero radial component of the surface velocity is assumed. Finally, following Champougny et al. [35], exchanges of surfactants with the bulk are neglected in the dynamics but accounted for in the apparent elasticity $E_{s}$ that can be measured independently in a Langmuir through for relevant interfacial velocities, and depends on the bulk concentration.

In this framework, and assuming axisymmetry along the axis aligned with gravity $g$, the drainage model consists in the following set of dimensionless evolution equations:

$$
\begin{aligned}
\frac{\partial h}{\partial t} & =-\frac{1}{\sin (\pi \theta)} \frac{\partial}{\partial \theta}\left[h \sin (\pi \theta)\left(u_{s}-\frac{h^{2}}{6} \frac{\partial p}{\partial \theta}\right)\right]-2 S t(1-\beta)\left(1+\frac{1}{\sqrt{t / \tau_{\mathrm{tr}}}}\right), \\
\frac{\partial p}{\partial \theta} & =\frac{1}{2} \frac{\partial}{\partial \theta}\left[\cos (\pi \theta)-\frac{B o}{\sin (\pi \theta)} \frac{\partial}{\partial \theta}\left(\sin (\pi \theta) \frac{\partial h}{\partial \theta}\right)+\frac{A}{h^{3}}\right], \\
h \frac{\partial p}{\partial \theta} & =B q(1+\Theta)\left[\frac{\partial}{\partial \theta}\left(\frac{1}{\sin (\pi \theta)} \frac{\partial}{\partial \theta}\left(\sin (\pi \theta) u_{s}\right)\right)\right]+2 B q \pi^{2} u_{s}-\frac{M a}{\Gamma} \frac{\partial \Gamma}{\partial \theta}, \\
\frac{\partial \Gamma}{\partial t} & =-\frac{1}{\sin (\pi \theta)} \frac{\partial}{\partial \theta}\left[\sin (\pi \theta)\left(\Gamma u_{s}-\frac{1}{P e_{s}} \frac{\partial \Gamma}{\partial \theta}\right)\right] .
\end{aligned}
$$

The polar coordinate $\theta$ has been scaled with $\pi$ and spans from $\theta=0$ at the south pole to $\theta=1$ at the north pole of the antibubble. The time $t$ has been scaled with $\tau_{0}=\mu_{g} \pi^{2} R /\left(\rho_{\ell} g h_{0}^{2}\right)$ corresponding to the viscousgravity timescale for drainage between rigid interfaces and where $\mu_{g}$ is the gas viscosity and $\rho_{\ell}$ is the liquid density. The gas shell thickness $h(\theta, t)$, the surface velocity $u_{s}(\theta, t)$, the surface concentration $\Gamma(\theta, t)$ and the pressure in the shell $p(\theta, t)$ have been scaled, respectively, with $h_{0}, \pi R / \tau_{0}, \Gamma_{0}$ and $p_{0}=2 \rho_{\ell} g R ; \Gamma_{0}$ being the initial concentration of surfactant at time $t=0$ and $p_{0}$ being the hydrostatic pressure difference driving the gas shell drainage. The parameter $\beta$ measures the degree of saturation of the gas in the liquid: $\beta=1$ corresponds to a fully saturated liquid, while $\beta=0$ corresponds to a fully degassed liquid. The other dimensionless parameters are defined as 
follow:

$$
\begin{array}{r}
S t=\frac{\pi^{2} \mu_{g} D_{g} S}{\rho_{\ell} g h_{0}^{3}}, \quad \tau_{\mathrm{tr}}=\frac{R^{2}}{\pi \tau_{0} D_{g}}, \quad B o=\frac{\gamma_{0} h_{0}}{\pi^{2} \rho_{\ell} g R^{3}}, \quad A=\frac{A^{\prime}}{6 \pi \rho_{\ell} g R h_{0}^{3}}, \\
B q=\frac{\eta_{s} h_{0}}{\pi^{2} \mu_{g} R^{2}}, \quad \Theta=\frac{\kappa_{s}}{\eta_{s}}, \quad M a=\frac{E_{s}}{\rho_{\ell} g R h_{0}}, \quad P e_{s}=\frac{\rho_{\ell} g R h_{0}^{2}}{\mu_{g} D_{s}},
\end{array}
$$

in which $D_{g}$ and $S$ are respectively the molecular diffusion coefficient and the solubility of the gas in the liquid, $\gamma_{0}$ is the equilibrium surface tension, $A^{\prime}$ is the Hamaker constant and $D_{s}$ is the surface molecular diffusion of surfactant molecules. The Stanton number $(S t)$ compares the air dissolution rate to the rate of drainage, the transient dimensionless time $\left(\tau_{\text {tr }}\right)$ compares the bulk diffusion timescale $R^{2} / \pi D_{g}$ to the drainage timescale, the Bond number $(B o)$ compares the capillary pressure to the hydrostatic pressure, the Hamaker number $(A)$ compares the disjoining pressure to the hydrostatic pressure, the Boussinesq number $(B q)$ compares the surface shear viscous stress to the bulk shear viscous stress in the gas shell, the ratio $(\Theta)$ compares the surface dilatational viscosity to the surface shear viscosity, the Marangoni number $(M a)$ compares the surface elasticity to the hydrostatic pressure force, and the surface Péclet number $\left(P e_{s}\right)$ compares the surface convection and the surface diffusion of surfactants.

To ensure symmetry with respect to the vertical axis, the set of equations (1) is solved with the following boundary conditions at the poles,

$$
u_{s}=\frac{\partial h}{\partial \theta}=\frac{\partial p}{\partial \theta}=\frac{\partial \Gamma}{\partial \theta}=0 \quad \text { at } \quad \theta=\{0,1\},
$$

Finally, uniform initial conditions for the thickness and the surface concentration are imposed, namely

$$
h=\Gamma=1 \quad \text { in } \quad t=0 .
$$

The model was implemented in Comsol using the PDE mode and a direct solver. The temporal solver uses the implicit backward differentiation formula (BDF) method at order 3 [36]. The number of elements was 3000 in the domain $\theta=[0,1]$ in order to ensure convergence, with mesh refinement near $\theta=0$ to capture the film destabilization due to the van der Waals instability (see [27] for details). Mesh-independence on the solutions was verified. 


\subsection{Parametric analysis}

Given the large number of dimensionless parameters associated to the various physical effects included in the modeling, one should restrict the parametric analysis to practical situations, and investigate some deviations of interest, essentially relative to surface elasticity $E_{s}$, saturation parameter $\beta$ and antibubble dimensions $R$ and $h_{0}$. Therefore, in the following, and unless specified otherwise, the parameter values used for solving the model are those given in Table 1. These parameters correspond to an antibubble with air for

Table 1: List of dimensional parameters used in the simulations and the associated dimensionless numbers. Note that $\tau_{0}$ and $p_{0}$ are calculated parameters.

\begin{tabular}{|l|c||c|c|}
\hline Dimensional & Value & Dimensionless & Value \\
\hline$R[\mathrm{~mm}]$ & 7 & $\tau_{\text {tr }}$ & 537 \\
$h_{0}[\mu \mathrm{m}]$ & 3 & $\beta$ & 1 \\
$\rho_{\ell}\left[\mathrm{kg} / \mathrm{m}^{3}\right]$ & 997 & $S t$ & $2.77 \times 10^{-2}$ \\
$g\left[\mathrm{~m} / \mathrm{s}^{2}\right]$ & 9.81 & $B o$ & $2.72 \times 10^{-6}$ \\
$\gamma_{0}[\mathrm{mN} / \mathrm{m}]$ & 30 & $A$ & $1.15 \times 10^{-6}$ \\
$E_{s}[\mathrm{mN} / \mathrm{m}]$ & 0.1 & $B q$ & $3.35 \times 10^{-4}$ \\
$\eta_{s}[$ Pa.s.m $]$ & $10^{-6}$ & $\Theta$ & 1 \\
$\kappa_{s}[$ Pa.s.m $]$ & $10^{-6}$ & $M a$ & 0.487 \\
$\mu_{g}[\mathrm{~Pa} . \mathrm{s}]$ & $1.85 \times 10^{-5}$ & $P e_{s}$ & 33307 \\
$A^{\prime}[\mathrm{J}]$ & $4 \times 10^{-20}$ & & \\
$D_{s}\left[\mathrm{~m}^{2} / \mathrm{s}\right]$ & $10^{-9}$ & & \\
$D_{g}\left[\mathrm{~m}^{2} / \mathrm{s}\right]$ & $2 \times 10^{-9}$ & & \\
$S[\mathrm{vol} / \mathrm{vol}]$ & 0.02 & & \\
$\tau_{0}[\mathrm{~s}]$ & 14.5 & & \\
$p_{0}[\mathrm{~Pa}]$ & 137 & & \\
\hline
\end{tabular}

the gas phase and, for the liquid phase, a Triton-X-100 surfactant solution at $10 \mathrm{CMC}$, with the critical micellar concentration $\mathrm{CMC}=0.02 \% \mathrm{w} / \mathrm{v}$. Triton$\mathrm{X}-100$, noted TX-100 hereafter, is non-ionic and has no measurable surface shear viscosity [30]. For the sake of simplicity $\eta_{s}$ and $\kappa_{s}$ have been fixed to $10^{-6}$ Pa.s.m, i.e. a one order of magnitude below the limit of resolution of the double wall ring (DWR) surface shear rheometer [37] that has been used as a consistency check Note that the model has been derived in the distinguished limit of a shear flow [38]. Consequently, exploring the limit of vanishing surface elasticity requires one to keep a residual surface viscous stress. Actually, the distinguished limit of an extensional flow cannot be 


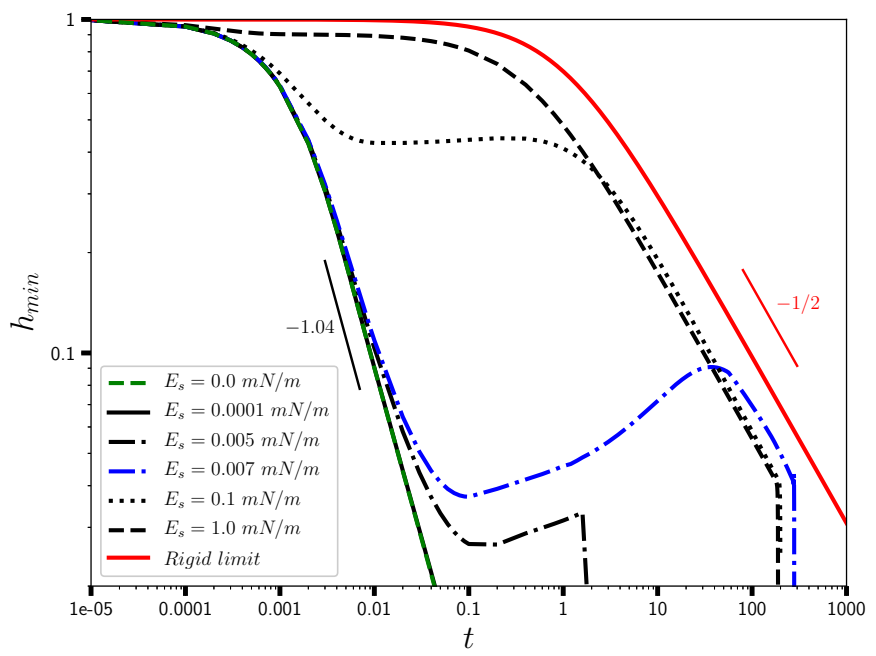

Figure 2: Time evolution of the minimum shell thickness near the south pole for several surface elasticities corresponding to $M a=\{0,0.0005,0.024,0.034,0.487,4.87\}$; see Table 1 for the other parameter values. The red line corresponds to the limiting case of an air shell draining between two rigid interfaces, as described by Eq. (5).

attained by the present model as the extensional viscous stresses in the bulk are negligible $\varepsilon^{2}$-order terms (see for instance [39] for hybrid model).

Additionally, the value of the surface diffusion coefficient has been estimated to be $D_{s}=10^{-9} \mathrm{~m}^{2} / \mathrm{s}$. The precise value has however no consequence on the numerical results as the surface Péclet number is already very high, namely $P e_{s} \gg 1$, which indicates a surfactant transport at the interface entirely governed by convection. Therefore the surface diffusion plays physically no role but is convenient for a numerical perspective, like an artificial diffusion. More importantly, the air shell of an antibubble being a closed system, the second-order diffusion term allows to impose the symmetry boundary conditions for the surface concentration $\Gamma$ at the two poles, as specified in (3).

\subsection{Numerical results}

As we are interested in the influence of the surface elasticity on the antibubble lifetime, Fig. 2 shows the time evolution of the minimum film thickness at the south pole for several values of the surface elasticity. For vanishing surface elasticity, $E_{s}=0.0001 \mathrm{mN} / \mathrm{m}$, the drainage is fast and essentially 
governed by the residual surface viscous stresses as no difference is observed with zero surface elasticity. In the drainage phase, the thinning of the shell at the south pole is $h_{\min } \propto t^{-1.04}$ and depends on the magnitude of the surface viscosities, as was shown in [27], the fact that the exponent is close to unity being a coincidence. The shell then thins until reaching the critical thickness for rupture due to van der Waals instability. As the elasticity is increased, $E_{s}=0.005 \mathrm{mN} / \mathrm{m}$, the Marangoni stress induced by the depletion of surfactants at the south pole of the antibubble counters the drainage before the film becomes thin enough to be destabilized by van der Waals forces. Interestingly, the behaviour of the thickness versus time is non-monotonic, and the minimum thickness increases as the Marangoni flow refills the air shell at the south pole, until concentration gradients weaken and the shell finally ruptures. At that moment, one can assume that the rupture is "instantaneous" as compared to the drainage time. The Marangoni effect is therefore found to drastically increase the lifetime of antibubbles above a surface elasticity between 0.005 and $0.007 \mathrm{mN} / \mathrm{m}$. The transition is thus sharp enough to define a threshold at $M a_{\mathrm{th}}=0.029 \pm 0.005$. Above this transition, the lifetime is two orders of magnitude larger and does not depend anymore on the surface elasticity. Indeed, the lifetimes for $E_{s}=0.1$ and $1 \mathrm{mN} / \mathrm{m}$ are comparable. In this regime, the drainage behavior approaches the limiting case of a film draining between two rigid interfaces, i.e. with no-slip conditions. This limit can be derived by setting $u_{s}=0$ in (1), together with $\beta=1$, and solving for spatially-independent thickness, i.e. $h=h(t)$, in the vicinity of the south pole, i.e. $\theta \rightarrow 0$, which gives

$$
h_{\min }=\frac{1}{\sqrt{1+\frac{\pi}{3} t}} .
$$

The striking feature is that for sufficiently high surface elasticity, the antibubble lifetime can drastically increase due to Marangoni stresses. In order to illustrate this, Fig. 3 presents the profiles of $h$ and $u_{s}$ for three values of the surface elasticity taken at representative times as indicated by the color scale. We observe that in the three cases, a gas pocket forms at the top of the antibubble as the gas accumulates due to drainage. However, the time at which this air pocket is prominent depends on the surface elasticity. For low surface elasticity, $E_{s}=0.0001 \mathrm{mN} / \mathrm{m}$, the surface velocity is always positive (see solid lines in (a) for $u_{s}$ ) and the lifetime is short. For high surface elasticity, $E_{s}=1 \mathrm{mN} / \mathrm{m}$, the Marangoni stress is such that the surface velocity changes sign (see dotted lines in (c) for $u_{s}$ ), meaning that the surface 

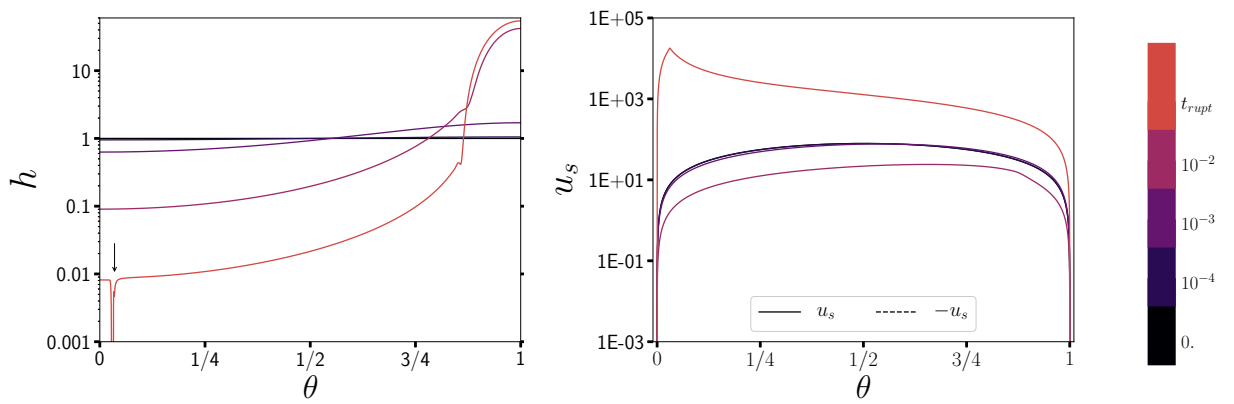

(a) $E_{s}=0.0001 \mathrm{mN} / \mathrm{m}$
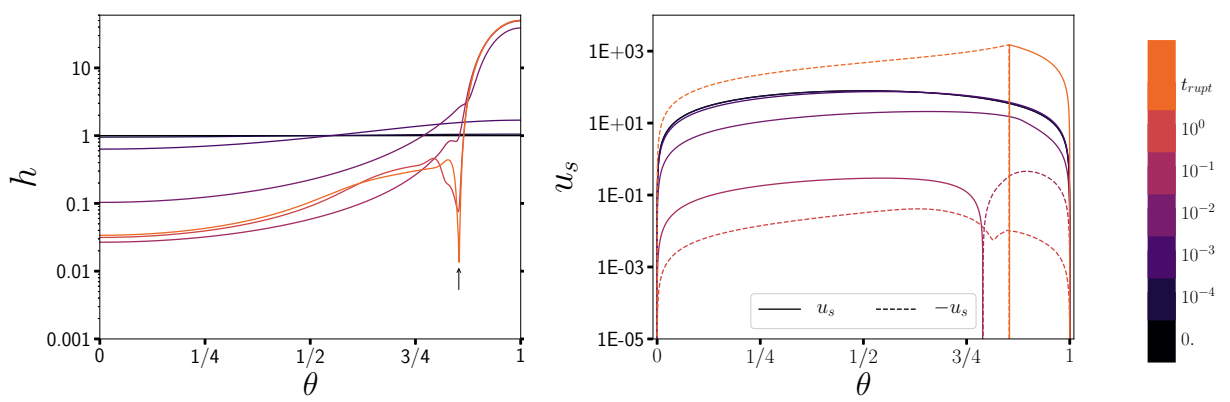

(b) $E_{s}=0.005 \mathrm{mN} / \mathrm{m}$
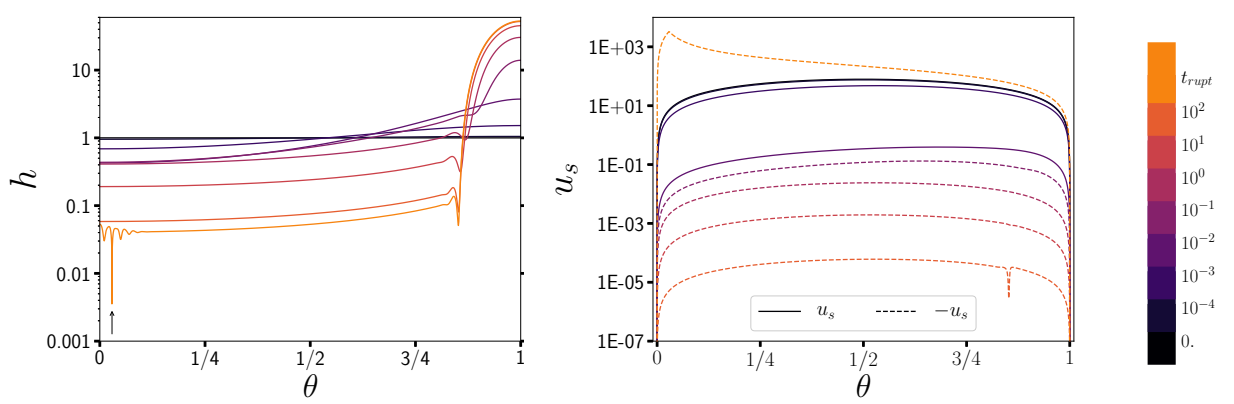

(c) $E_{s}=1 \mathrm{mN} / \mathrm{m}$

Figure 3: Time-evolution of the thickness and surface velocity profiles along the polar coordinate for three different elasticities corresponding to $M a=\{0.0005,0.024,4.87\}$; see Table 1 for the other parameter values. The rupture position is near the south pole for (a) and (c) and at the capillary ripple near the north pole for (b). Arrows indicate the rupture location (see text for details). 


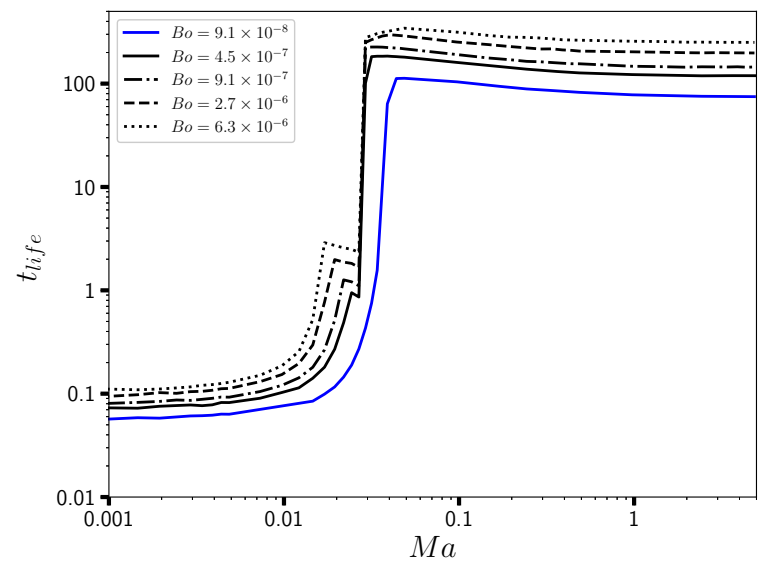

Figure 4: Antibubble lifetime in function of the Marangoni number for several surface tension $\gamma_{0}=\{1,5,10,30,70\} \mathrm{mN} / \mathrm{m}$; see Table 1 for the other parameter values.

flow opposes to the drainage and refills the shell at the south pole of the antibubble. For an elasticity around the transition, $E_{s}=0.005 \mathrm{mN} / \mathrm{m}$, surface velocity changes sign in time and in space (see solid and dashed lines in (b) for $u_{s}$ ). Interestingly enough for this case, the destabilization due to disjoining pressure occurs at the minimum thickness in the capillary ripple formed at the basis of the gas pocket, while for the other cases, the destabilization of the film occurs at the vicinity of the south pole. The rupture location in turn can have an impact on the lifetime, and especially at the transition, as shown in Figure 4 where the lifetime, denoted $t_{\text {life, }}$, is plotted versus the Marangoni number for different Bond numbers. The sharp transition in the lifetime at $M a_{\mathrm{th}} \approx 0.03$ presents a discontinuity corresponding to the jump in the rupture location: (i) at the south pole on the left of the discontinuity, as in Fig. 3a, and (ii) at the capillary ripple near the north pole on the right of the discontinuity, as in Fig. 3b; the capillary ripple triggering the van der Waals instability. On the two plateau's before and beyond the transition, the rupture occurs essentially at the south pole as in Fig. 3a,c. Now, when decreasing the Bond number, hence the surface tension, the amplitude of the capillary ripple is less pronounced and the rupture rather occurs essentially at the south pole. For $B_{o}=9.1 \times 10^{-8}$ (blue line in Fig. 4), the discontinuity is even absent. Finally the lifetime increases with increasing surface tension, as capillary forces oppose to van der Waals forces in the destabilisation mechanism leading to rupture. 


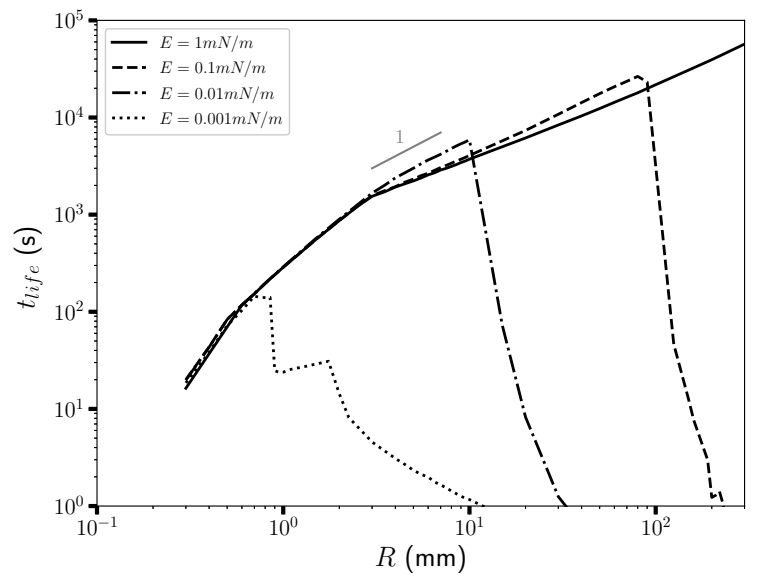

Figure 5: Dimensional antibubble lifetime as a function of the radius for various surface elasticities; see Table 1 for the other parameter values. The slope ' 1 ' corresponds to the limit of rigid interfaces, i.e. $t_{\text {life }} \propto R$ as it can be deduced from Eq. (5).

With the aim to compare the numerical results with experimental ones in section 4, we plot in Fig. 5 the dimensional lifetime versus the radius of antibubbles for various surface elasticities. For sufficiently small antibubbles, surface elasticity can be strong enough to oppose to the drainage and delay drastically the lifetime. Now, for a given elasticity, there is always a radius beyond which the Marangoni effect is not strong enough to counteract the drainage, whose strength is proportional to the radius $R$ measuring the hydrostatic pressure difference. The Marangoni number indeed compares the two effects. Nevertheless, for sufficiently large surface elasticity, i.e. $E_{s}=1 \mathrm{mN} / \mathrm{m}$, the Marangoni effect dominates the drainage over a wide range of antibubble radii. The Marangoni number does play a similar role here than the 'rigidity' parameter introduced in the context of film formation by Champougny et al. [35]. Above the transition value, the interfaces are rigidified by the Marangoni stress, while below this transition, there is a loss of rigidity that results in the impossibility of making long-living antibubbles, exactly like in the formation of soap films [35]. This loss of rigidity appears at $M a_{\text {th }} \lesssim 0.03$, as was shown in Fig. 4 . Note that the discontinuity in the curve for $E_{s}=0.001 \mathrm{mN} / \mathrm{m}$ in Fig. 5 is again due to a sudden change of rupture location. In other words, the change in interfacial mobility and motion is causing changes in the evolution of the thickness profile, leading, among other things, to a change in rupture location. 
Table 2: Wide dispersion of antibubble lifetimes in literature experiments showing no clear trend with the antibubble radius.

\begin{tabular}{|cccc|}
\hline Surfactant & Radius $(\mathrm{mm})$ & Lifetime $(\mathrm{s})$ & Ref. \\
\hline Dawn $^{\text {TM }}$ Ultra $+\mathrm{NaCl}$ & $3-11$ & $5-60$ & {$[21]$} \\
$\mathrm{C}_{12} \mathrm{E}_{6}$ at $10 \mathrm{CMC}$ & $3-15$ & $5-700$ & {$[18]$} \\
\hline
\end{tabular}

\section{Experiments}

The clear dependence of the antibubble lifetime on the radius found in Fig. 5 does not match the previous experimental observations [18, 21] showing no trend, possibly hidden by a wide dispersion of lifetimes as compiled in Table 2. Among the various potential sources of dispersion, there is the way antibubbles were made in previous studies, namely by hand, preventing their controlled production. We therefore designed an antibubble generator that allows to produce on-demand antibubbles in a reproductive manner and by controlling the gas phase. Next we briefly present the antibubble generator (see details in [32] and video in [40]), the materials and methods and the surfactant characterization.

\subsection{Antibubble generator}

The system shown in Fig. 6 consists of an immersed bell into a liquid bath, an injector tube, a pressure controller (Fluigent MFCS ${ }^{\mathrm{TM}}-\mathrm{EZ}$ ) to deliver the fluids and two solenoid valves (Fluigent 2-SWITCH ${ }^{\mathrm{TM}}$ ) to dispense the liquid and gas on demand. The gas pressure is adjusted to maintain the position of the gas-liquid interface at the bottom of the bell. The liquid inlet produces a jet inside the bell through the injector. Figure 7 shows the time sequence for producing antibubbles. At about $60 \mathrm{~ms}$ after the liquid injection, the jet penetrates the gas-liquid interface entraining a thin gas shell. This gaswrapped jet destabilizes and pinches-off by the Rayleigh-Plateau instability [10] at around $180 \mathrm{~ms}$. Once formed, it takes about $250 \mathrm{~ms}$ for the capillary oscillations to be damped and the antibubble to become spherical. The whole formation process takes less than half a second. Note the liquid injection needs to be interrupted using the valves before making the next antibubbles, such as to recreate a gas shell.

As compared to hand-made antibubbles [10], this set-up allows a much finer control of the various parameters influencing the formation, such as the 


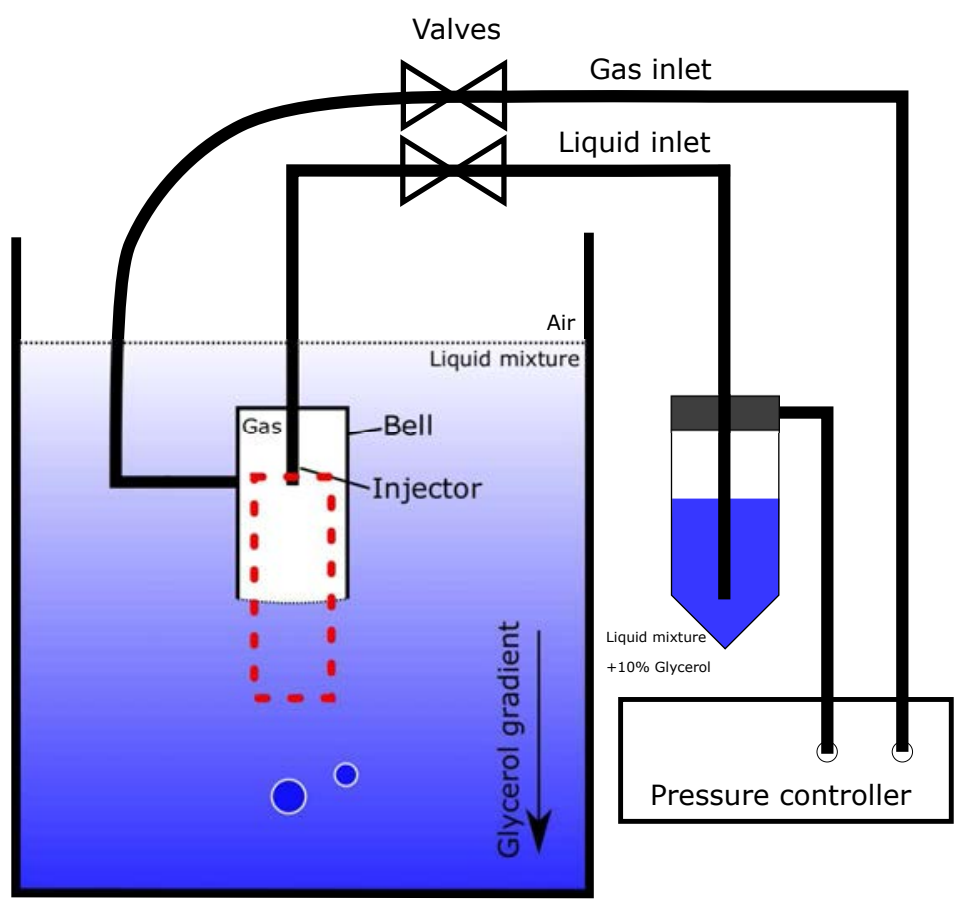

Figure 6: On-demand antibubble generator. The tank size is $10 \times 10 \times 10 \mathrm{~cm}^{3}$, the bell's size is $4 \mathrm{~cm}$ in height and $1 \times 1 \mathrm{~cm}^{2}$ in cross section, the internal diameter (ID) of the injector is $0.7 \mathrm{~mm}$ and the distance between the injector and the interface is about $2 \mathrm{~cm}$. The dashed red rectangle indicates the observation area for the time sequence of Fig.7 .

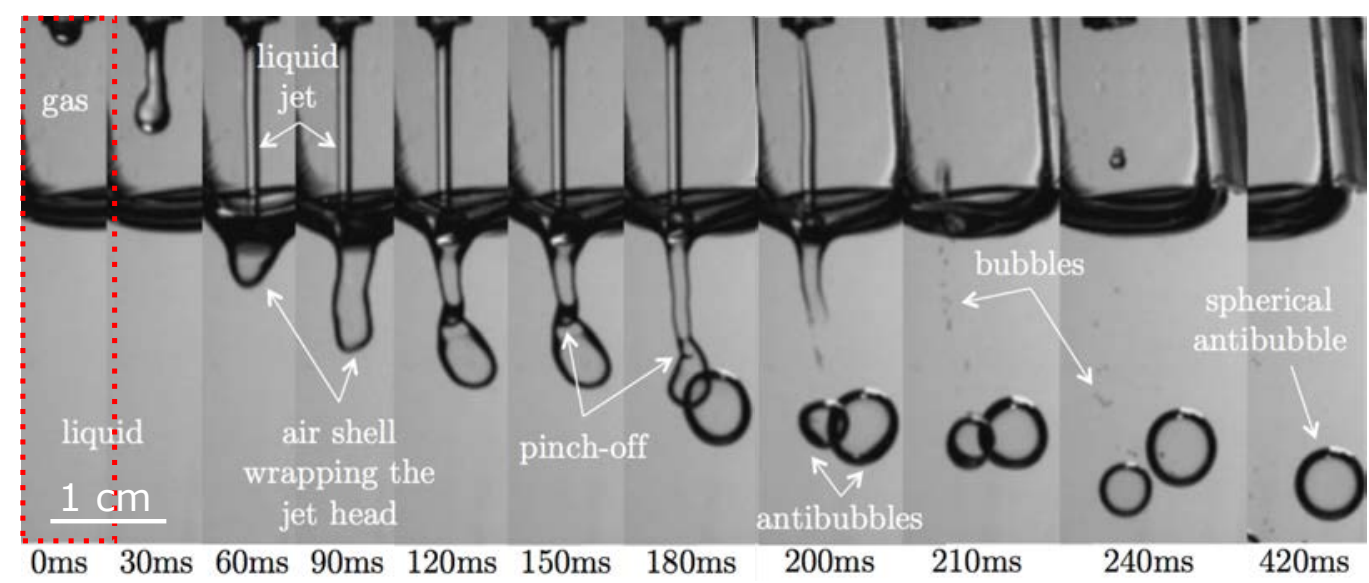

Figure 7: Time sequence of antibubble formation (200 fps, exposure time: $1 \mathrm{~ms}$ ). Note that the width of each frame size is different. The dashed red rectangle corresponding to the observation area, as schematized in Fig. 6, measures $0.8 \times 4.6 \mathrm{~cm}^{2}$. 
diameter of the tip of the liquid injector, the distance between this tip and the gas-liquid interface, the velocity of the injected liquid jet and the nature of the gas. More specifically, two internal diameters (ID) of the injector were used in this study to increase the range of antibubble radius: (i) for $\mathrm{ID}=0.7 \mathrm{~mm}$, the range is $R=[0.5,2.5] \mathrm{mm}$, and (ii) for $\mathrm{ID}=1.6 \mathrm{~mm}$, the range is $R=[1,4] \mathrm{mm}$. The distance of the injector with the gas-liquid surface at the bottom of the gas bell was also adapted, namely (i) $d=20 \mathrm{~mm}$, and (ii) $d=25 \mathrm{~mm}$.

In order to minimize the external perturbation on antibubbles, they were sank by adding $10 \%$ vol of glycerol to the surfactant mixture used to form the core. To prevent the antibubble to collapse when reaching the bottom of the tank, a gradient of glycerol was created inside the bath. Consequently, the antibubble sank until reaching the neutrally buoyant position located between two to four centimetres above the bottom, depending on their size and air shell thickness. With a variation of less than $0.2 \%$, the concomitant pressure, and hence solubility, differences are small enough to have no significant influence on the antibubble lifetime.

\subsection{Materials and Methods}

In the present study, Triton X-100 (TX-100) and $\mathrm{C}_{12} \mathrm{E}_{6}$ surfactants were used to produce antibubbles. Triton X-100 (purity $\geq 99 \%$ ), $\mathrm{C}_{12} \mathrm{E}_{6}$ (purity $\geq 98 \%$ ) and glycerol (purity $\geq 99 \%$ ) were purchased from Sigma-Aldrich@). Surfactants were diluted in ultra-pure water obtained by an Arium UV PRO (Sartorius $\mathrm{R})$ ) (conductivity $\leq 0.055 \mu \mathrm{S} / \mathrm{cm})$. Filtered and oil-free compressed air was used to fill the bell of the antibubble generator and pressurize the liquid injector. Before each experiment the entire set-up was cleaned with Isopropyl alcohol and thoroughly rinsed with ultra-pure water. A Fibox 4 probe (Presens $\AA$ ) was used to measure the saturation of Oxygen in the bath assuming that the measurement of Oxygen saturation corresponds to air saturation in water in standard conditions [41]. The liquid was bubbled during several hours before each set of experiments. A saturation value between $96 \%$ and $98 \%$ with an accuracy of $2 \%$ was measured during experiments at the periphery of the reservoir such as the probe did not disturb the antibubble formation.

Antibubbles were observed using an Y3 high speed camera (IDT Vision@) with a pixel size of $10.85 \mu \mathrm{m}$ and acquisition frequency of $30 \mathrm{~Hz}$, which allow measuring accurately their sizes with a resolution of $80 \mu \mathrm{m}$ on the diameter and their lifetimes with a resolution of $70 \mathrm{~ms}$, taken from their generation 


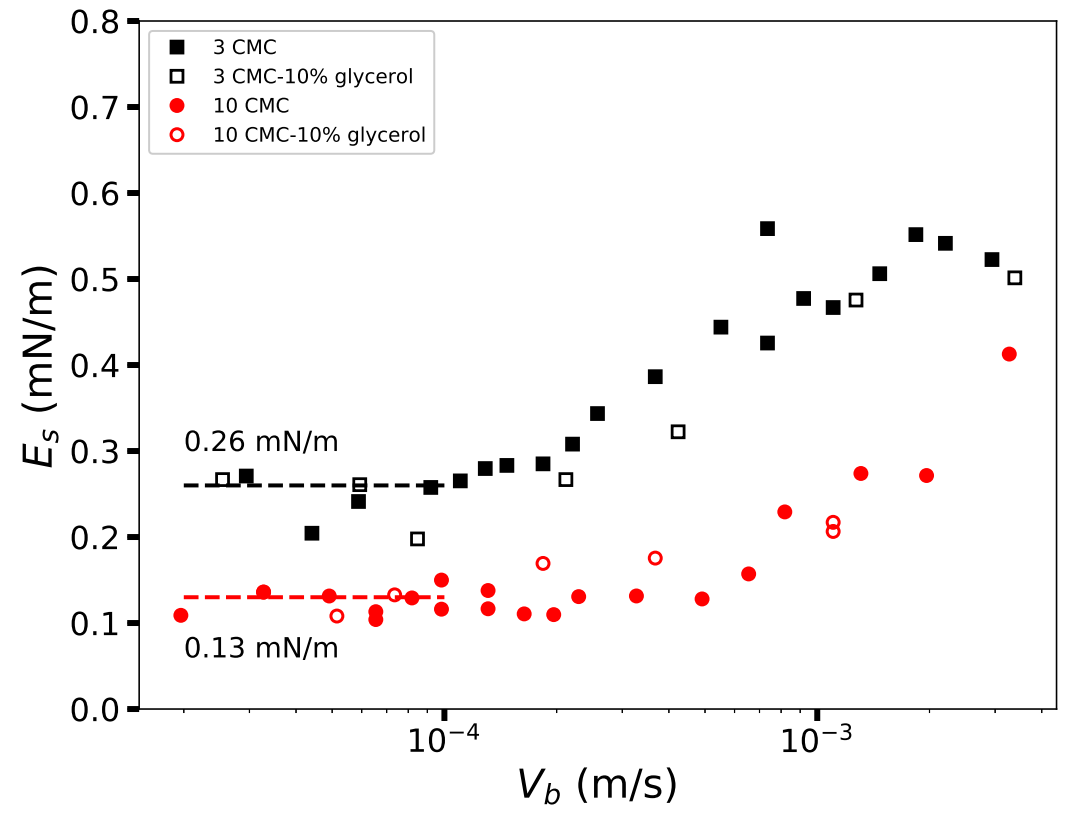

Figure 8: Surface elasticity measurements using a Langmuir trough for various barrier speeds $V_{b}$. Increasing the concentration of TX-100 decreases the surface elasticity. The glycerol has no significant influence on the surface elasticity.

to their collapse. All antibubble were observed from the bottom of the tank, except images on Fig. 7 taken form the side (see dashed red rectangle) and $1000 \mathrm{~Hz}$. All experiments were conducted in an ISO 7 clean room at a temperature of $20^{\circ} \mathrm{C}$, unless specified otherwise.

\subsection{Surfactant characterization}

In order to compare experimental data with the model, the surfactant mixture properties are required. Surface elasticity and density were measured for mixtures of TX-100 at 3,5 and $10 \mathrm{CMC}$ with and without glycerol. Elasticity of TX-100 was measured using a Langmuir through (model KN 1003 from Biolin Scientific $囚$ ) and following the protocol of Champougny et al. [35] consisting in measuring the time evolution of the surface tension $\gamma(t)$ while the barriers at the liquid interface are moved apart at constant speed $V_{b}$, starting with the equilibrium surface tension $\gamma_{0}=\gamma(t=0)$. Figure 8 presents the elasticity of TX-100 for several barrier speeds. Similar to the results of [35] for $\mathrm{C}_{12} \mathrm{E}_{6}$, surface elasticity decreases with an increase of the 
bulk concentration of TX-100. We recall here that the surface elasticity is modelled as if the surfactant was insoluble, even though exchanges between the surface and the bulk are present. We thus believe that the decrease of this "effective" surface elasticity is a consequence of the decrease in excess surface concentration. It is observed that the presence of glycerol does not affect the elasticity measurements at both concentrations of TX-100. Notice that for speeds corresponding to antibubble drainage dynamics, i.e. $V_{b} \leq 10^{-4} \mathrm{~m} / \mathrm{s}$, the surface elasticity is independent of $V_{b}$, as illustrated by the dashed lines, which allows us to use a mean value for each concentrations of TX-100 used. Liquid densities are determined by measuring the weight of a given volume. These measurements are reported in Table 3.

Table 3: Properties of surfactant mixtures at $25^{\circ} \mathrm{C}$ used in antibubble experiments. Mixture with $10 \%$ vol of glycerol have similar values to those without glycerol except for density.

\begin{tabular}{llll}
\hline Mixture & $\gamma_{0}(\mathrm{mN} / \mathrm{m})$ & $E_{s}(\mathrm{mN} / \mathrm{m})$ & $\rho_{\ell}\left(\mathrm{kg} / \mathrm{m}^{3}\right)$ \\
\hline $\mathrm{C}_{12} \mathrm{E}_{6}$ 10 CMC & $32.0[42]$ & $0.18[35]$ & $997 \pm 2$ \\
TX-100 3 CMC & $30[43]$ & 0.13 & $995 \pm 2$ \\
TX-100 3 CMC with glycerol & 30 & 0.13 & $1018 \pm 2$ \\
TX-100 10 CMC & $30[43]$ & 0.26 & $995 \pm 2$ \\
TX-100 10 CMC with glycerol & 30 & 0.26 & $1018 \pm 2$
\end{tabular}

Note that the measured surface elasticities are much smaller by at least two orders of magnitude than the Gibbs elasticity reported by Wantke et al. [44] for Triton-X-100. Nevertheless, these elasticities correspond to surfactant concentrations much below the CMC by at least one order of magnitude. They also have been obtained for strain rates much larger than $1 \mathrm{~Hz}$. On the contrary, and similarly to Champougny et al. [35] for $\mathrm{C}_{12} \mathrm{E}_{6}$, the strain rates in our measurements are below $0.1 \mathrm{~Hz}$ and the surfactant concentrations are much above the CMC, which explains why the effective elasticities are much below the values in [44], essentially because it is strongly affected by mass transfer effects between the bulk and the interface.

\subsection{Liquid viscosity}

The bulk liquid viscosity of the TX-100 mixture without glycerol has been measured with a HAAKE ${ }^{\mathrm{TM}}$ Falling Ball Viscometer type $\mathrm{C}$ (Thermofisher scientific@), which lead to $\mu_{\ell}=0.908 \mathrm{mPa}$.s at $25^{\circ} \mathrm{C}$ and for a $10 \mathrm{CMC}$ concentration. Note that the liquid viscosity is only needed in section 4.2 
to estimate the initial film thickness from the rising velocity of antibubbles. Otherwise, the liquid bulk viscosity is not involved in the modelling since in the context of lubrication approximation, the viscous shear at the interfaces from the liquid phase is negligible as compared to the viscous shear from the gas phase, as long as $\mu_{\ell} / R \ll \mu_{g} / h_{0}$. This inequality is still verified with a $10 \%$ glycerol solution as the viscosity remains of the same order of magnitude, namely $1.21 \mathrm{mPa.s}[45,46]$. Therefore, the increase of viscosity due to the presence of glycerol in the liquid phase has been assumed in this work to have no impact on the antibubble lifetime.

\section{Results}

In this section, we aim at unravelling the wide dispersion of antibubble lifetimes observed experimentally by sequentially sounding the various possible sources of dispersion, in light with the theoretical model of section 2.1.

\subsection{Influence of surface elasticity}

The first set of experiments have been realized with a mixture of TX-100 at different concentrations to probe the influence of the surface elasticity on the antibubble's lifetime. Figure 9 presents the lifetime for three concentrations and for radii ranging from 0.5 to $2.5 \mathrm{~mm}$. During experiments, some antibubbles collapsed when they contact another antibubble or any solid part of the system. Those premature deaths are not related to the drainage dynamic of the gas shell and have then been disregarded from the experimental data.

Firstly, Fig. 9 shows no influence of the concentration of surfactant on the antibubble lifetime. This is in accordance with the numerical results of Fig. 4 showing that, above a threshold value of the Marangoni number, the lifetime remains constant for a fixed equilibrium surface tension. Considering the surface elasticity reported in Table 3, and for the given range of radius, the Marangoni number is always larger than unity, i.e. much larger than $M a_{\text {th }} \sim 0.03$ below which the interfaces loose their rigidity.

Secondly, experimental results demonstrate a clear dependence of antibubble's lifetime on the radius, a trend that has never been reported experimentally before, even though it is supported by classical drainage theory (see for instance [47]) and actually in agreement with our numerical results plotted in Fig. 5. 


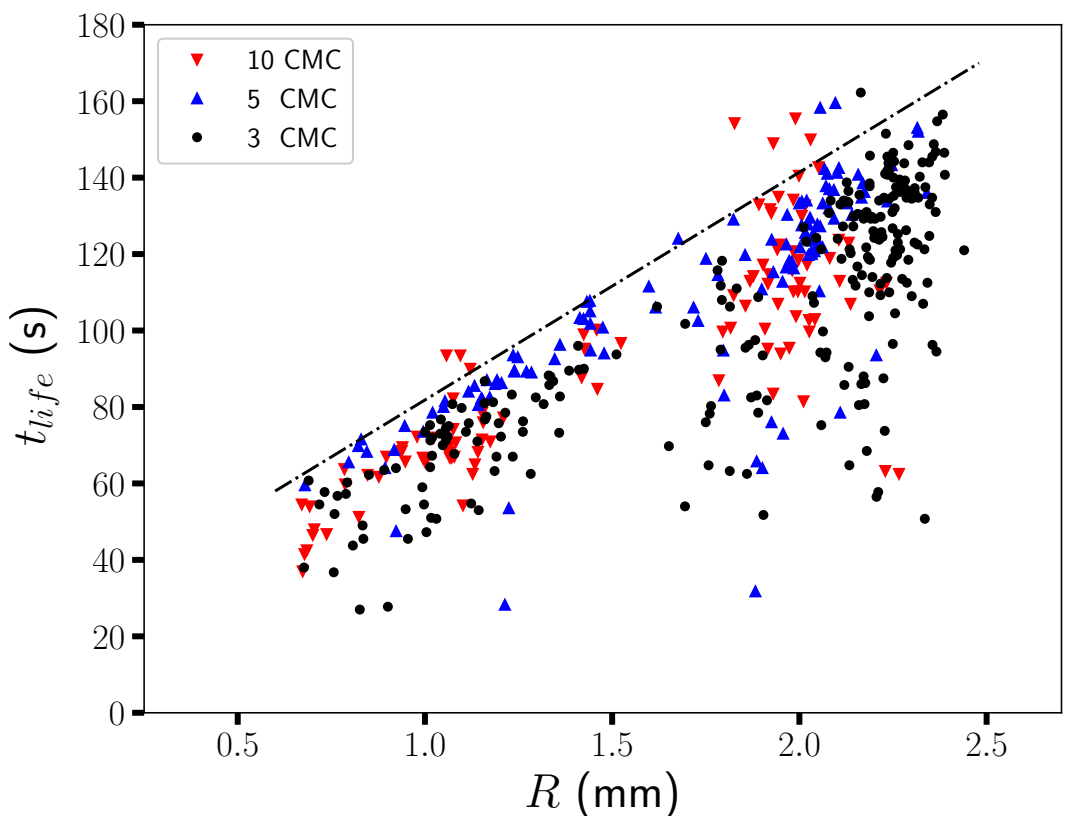

Figure 9: Antibubble lifetime depending on the radius; mixture of water with TX-100 at 3, 5 and $10 \mathrm{CMC}$ with $10 \% \mathrm{vol}$ of glycerol. The dot-dashed line guides the eyes to emphasize the radius dependency of the lifetime. 
Despite the good qualitative agreement with the model, the experimental results depict a large dispersion that prevents any quantitative comparison and therefore needs further inspection. Since the three concentrations investigated in Fig. 9 are not discriminating in terms of surface elasticity, the three corresponding sets of data are represented as a single set in the following and the mean value of surface elasticity extracted from Fig. 8, mainly $E_{s}=0.2 \mathrm{mN} / \mathrm{m}$, is fixed hereafter for further comparison with the model.

\subsection{Influence of initial gas thickness}

Prior to any quantitative comparison with the model, one needs to evaluate the potential source of dispersion originating from the dispersion of the initial thickness of the gas shell. Actually, despite the automatic antibubble generation, the gas-liquid interface is continuously deformed by surface waves produced by previous generated antibubbles. Consequently, variability exists in the way the liquid jet impinges the gas liquid interface and entrains the gas, which can in turn influence the initial gas shell thickness. An estimation of this initial shell thickness can be obtained with the measurement of the terminal velocity of rising antibubbles [21], provided no glycerol is used. The terminal velocity results from the balance between the buoyancy force, due to the density difference of the antibubble compared to the surrounding liquid, and the drag force. By considering that $h_{0} / R \ll 1$, this balance writes

$$
\frac{4}{3} \pi R^{3}\left(\rho_{\ell}-\rho_{\mathrm{AB}}\right) g=\frac{1}{2} \pi R^{2} \rho_{\ell} C_{D} V_{t}^{2},
$$

where $\rho_{\mathrm{AB}}$ is the density of the antibubble, $C_{D}$ is the drag coefficient and $V_{t}$ is the terminal velocity of the rising antibubble. Neglecting the gas density as compared to the liquid density, the density difference can be approximated in turn by

$$
\rho_{\ell}-\rho_{\mathrm{AB}}=\frac{3 h_{0}}{R} \rho_{\ell} .
$$

Combining Eqs (6) and (7) yields an expression for the initial film thickness

$$
h_{0}=\frac{C_{D} V_{t}^{2}}{8 g} \text {. }
$$

Similarly to the study of Kim and Vogel [21], the antibubble is considered as a rigid sphere, which is supported by a large Marangoni number corresponding to high surface rigidity. This was also observed by Matsumoto et al. [48] 
who measured the terminal velocity of a bubble in a mixture with a small amount of Triton-X-100 ( $c \ll \mathrm{CMC}$ ), and found drag coefficients coinciding perfectly with the one of a rigid sphere in the range $50<R e<90$. Here also, and contrarily to [21], we consider the corresponding drag coefficient in the intermediate range of Reynolds numbers, as encountered in our experiments, i.e. $5<R e<200$, with $R e=2 \rho_{\ell} V_{t} R / \mu_{\ell}$. According to Allen's drag model [49], an approximate drag coefficient for a rigid sphere in this range is

$$
C_{D} \approx 18.5 R e^{-3 / 5}
$$

which matches within maximum $15 \%$ difference the more accurate correlation of Turton and Levenspiel [50] as shown in [51]. Plugging Eq. (9) into Eq. (8) finally gives

$$
h_{0} \approx \frac{1.526}{g} \sqrt[5]{\frac{\mu_{\ell}^{3} V_{t}^{7}}{R^{3} \rho_{\ell}^{8}}} .
$$

Figure 10 presents the measurements of $h_{0}$ obtained with Eq. (10) using the observable $V_{t}$, for 130 rising antibubbles with radii ranging between 0.5 and $4 \mathrm{~mm}$. The mean initial gas shell thickness is $h_{0_{\text {mean }}}=4 \mu \mathrm{m}$ with a standard deviation of $\pm 1.3 \mu \mathrm{m}$. The inset of Fig. 10 shows that for small antibubbles (red disks), the terminal velocity increases with $R$, while it is rather constant for larger antibubbles (green triangles). The explanation can be found in the formation mechanism of the antibubble, which relies on the Landau-Levich-Derjaguin law, as exposed in Appendix A. As inferred from the set of Eqs. (A.1-8), if the radius $a$ of the penetrating jet is smaller than the capillary length $\ell_{c}, h_{0}$ strongly depends on $R$, otherwise it does much less. This is illustrated by the solid line in Fig. 10, showing a compatible trend with the data even though the dispersion remains important. This is mainly due to the variability of the velocity $(U)$ of the impacting jet that can vary in a wide range, i.e. $0.1<U<0.4 \mathrm{~m} / \mathrm{s}$, and has not been systematically measured as it requires a specific set-up. To a lower extend, selected wavelength parameter $(\alpha)$ of the instability, can also vary and depends on the jet impact, as outlined in Appendix A. Few extreme values are also observed in Fig. 10, up to $h_{0}=10 \mu \mathrm{m}$, which can be due to flow movements in the bulk liquid or to interactions with other antibubbles. Without clear causality, all points were taken into account in the normal distribution fitting the data. Now the mean value of $4 \mu \mathrm{m}$ is in good agreement with values found in literature with other 


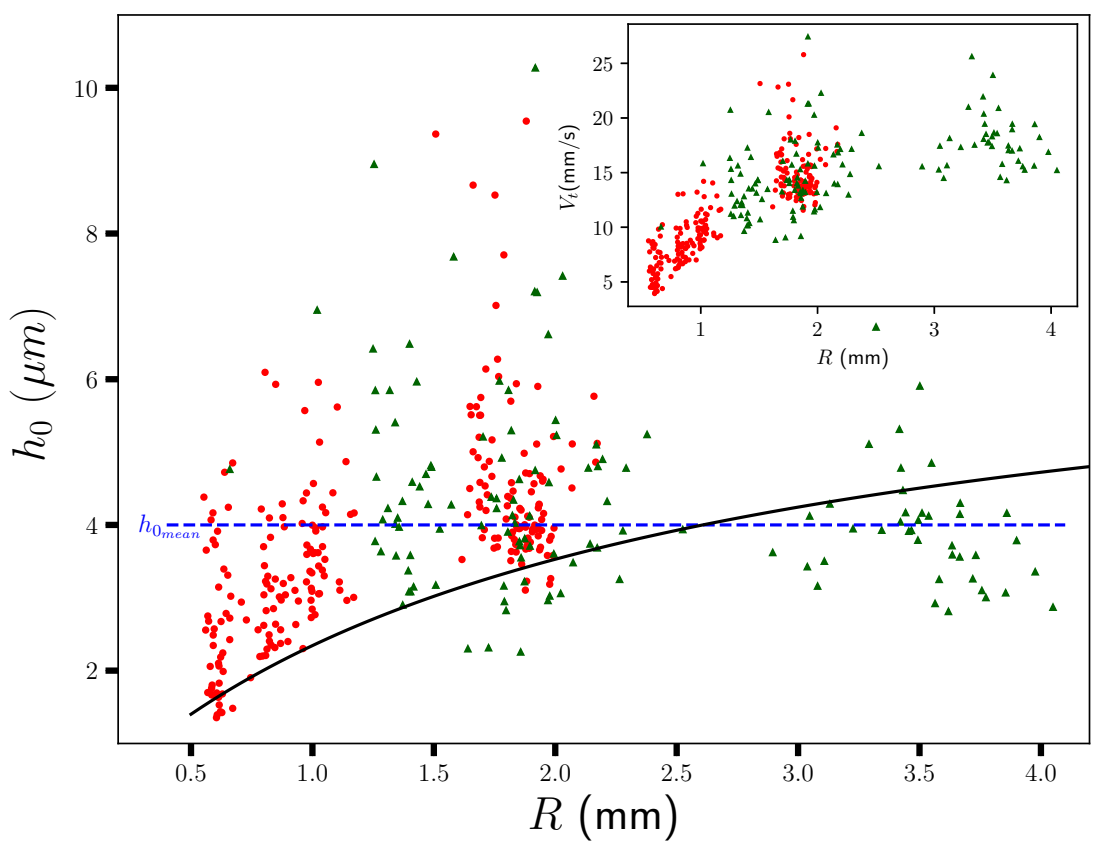

Figure 10: Initial gas thickness obtained with Eq. (10) and using measurement of the terminal velocity $V_{t}$ (see inset) for rising antibubbles in a mixture without glycerol and with TX-100 at concentration 10 CMC. Red disks and green triangles are data obtained with the $0.7 \mathrm{~mm}$ and $1.6 \mathrm{~mm}$ ID injector, respectively. The solid line is the theoretical prediction outlined in Appendix A. 


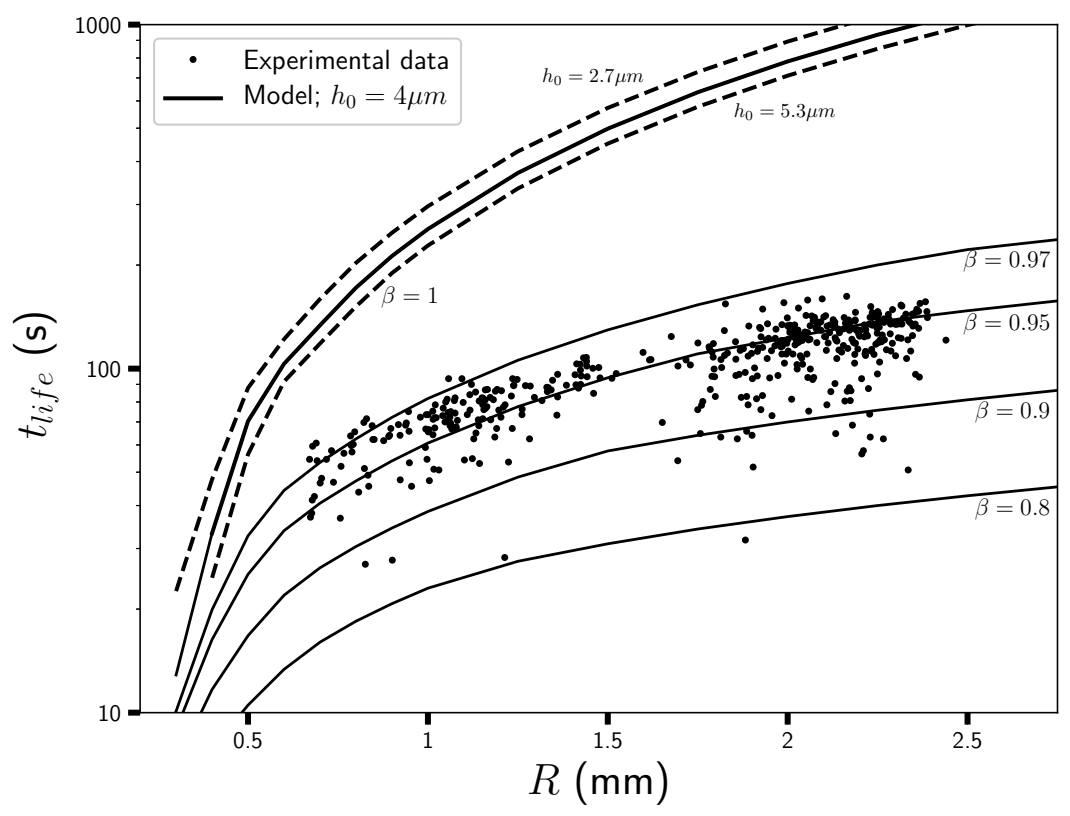

Figure 11: Influence of initial gas thickness $h_{0}$ and saturation parameter $\beta$ on antibubble lifetime and comparison with experimental data; for the numerical simulations, $E_{s}=$ $0.2 \mathrm{mN} / \mathrm{m}$ and $\eta_{s}=\kappa_{s}=10^{-6}$ Pa.s.m. Solid line represents simulations obtained with $h_{0}=4 \mu \mathrm{m}$, while the dashed lines are obtained with $h_{0}=4 \pm 1.3 \mu \mathrm{m}$, corresponding the standard deviation of the thickness measurements.

techniques, such as for instance $h_{0}=3.2 \mu \mathrm{m}$ using the gas dissolution time as an observable [31], or $h_{0}=5 \mu \mathrm{m}$ using the volume of the air bubble formed after the antibubble collapse [19].

Next, the measured value of $h_{0}$ is used in the model to compare with our experimental data as reported in Fig. 11. We observe a large difference between the theoretical and experimental lifetimes that cannot be explained by the dispersion of the initial gas thickness $( \pm 1.3 \mu \mathrm{m})$, as delimited by the dashed lines. Note in addition that a larger initial gas thickness leads to a lower antibubble's lifetime, which is counter-intuitive. It can be explained by the larger velocity of the gas in the shell during the early viscous drainage phase, i.e. before the Marangoni effect settles down and opposes to the drainage.

As no significant influence of the initial gas shell thickness has been obtained, even with a large dispersion, we keep the value fixed to the mean 
value $h_{0}=4 \mu \mathrm{m}$ in the following and look for other effects.

\subsection{Influence of gas saturation}

A possible explanation for the lower antibubble's lifetime observed experimentally is the undersaturation of the bulk liquid with gases that leads to the partial dissolution of antibubble gas shell [31]. Indeed, despite the special care we took in having a bulk saturated in gas, we measured slightly under-saturated values, i.e. $\beta<1$, with our oxygen probe, even though by only few percents. Yet, we compare in Fig. 11 the experimental lifetimes with numerical predictions computed for different gas saturation parameters $\beta$.

The model predicts a significant decrease of the lifetime for only few percents of undersaturation, hence in the limit of resolution of the oxygen probe. These results demonstrate the importance of controlling the gas content in the liquid when making antibubbles, as already reported in [31]. A key point here is that despite the uncertainty on the absolute value of the gas saturation, it was found to be very stable in time, within $2 \%$. However, to encompass the shortest lifetime, one would had to set $\beta=0.8$, which is much below the minimum value of saturation measured experimentally, namely $\beta=0.94$ accounting for $2 \%$ accuracy. Consequently, even though quantitative agreement can finally be obtained with most of the experimental data when accounting for partial gas dissolution, it still does not explain the wide dispersion of the antibubble lifetime, and especially for the shortest lifetimes.

In what follow, we fix the saturation parameter to the upper bound in Fig. 11, i.e. $\beta=0.97$, and focus on the rupture mechanism.

\subsection{Influence of dust particles}

The model assumes that the gas shell is destabilized by van der Waals interactions when the film thickness becomes smaller than about $100 \mathrm{~nm}$ [52]. What if instead the film prematurely breaks because of the presence of dust particles in the gas phase? This mechanism has been detailed by Denkov et al. [53] who analyzed dust particles as antifoam "compounds". EvangelioSànchez [54] has recently demonstrated the crucial role of dust particles in the premature collapse of floating bubbles. We account for this phenomenon into the model by imposing an arbitrary critical thickness $h_{c}$ at which an antibubble collapses. Figure 12 presents the theoretical lifetime for three different critical thicknesses $h_{c}=0.2,0.3$ and $0.5 \mu \mathrm{m}$, in addition to the 


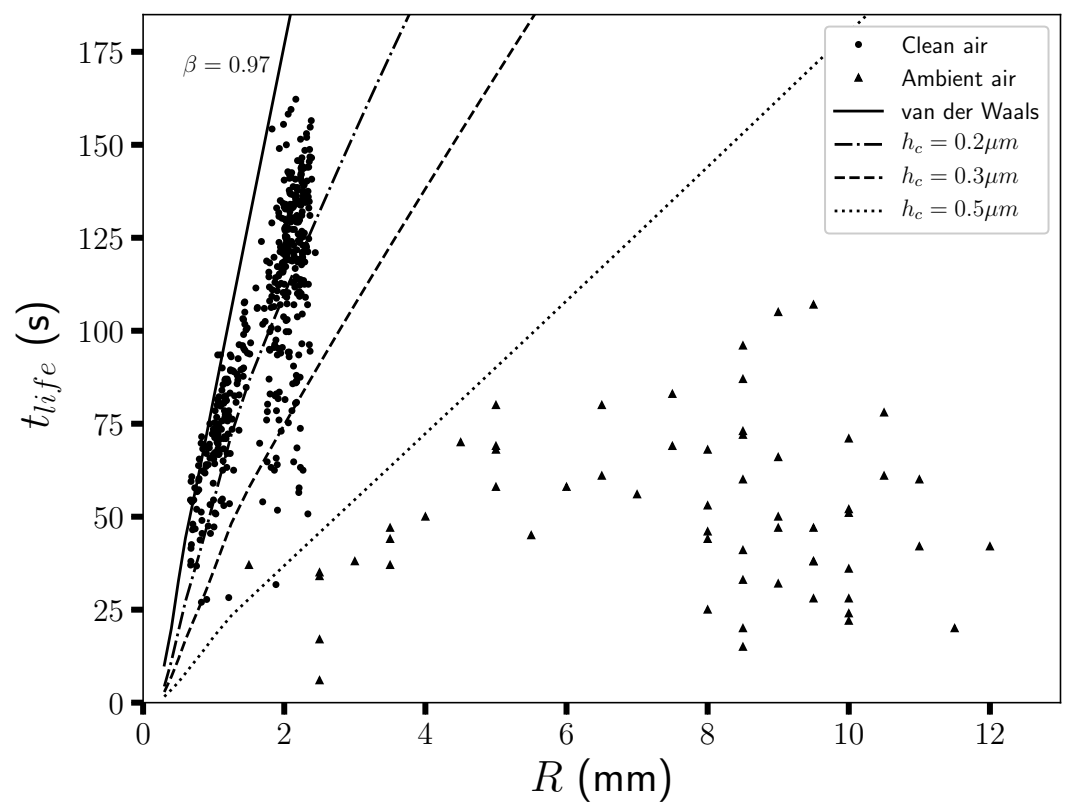

Figure 12: Comparison of experimental lifetime with numerical simulations for several critical thicknesses $h_{c}$. For the numerical simulations, $E_{s}=0.2 \mathrm{mN} / \mathrm{m}, \eta_{s}=\kappa_{s}=$ $10^{-6}$ Pa.s.m, $h_{0}=4 \mu \mathrm{m}$ and $\beta=0.97$. Experimental data come from two data set obtained with TX-100 mixture: a set obtained with the antibubble generator in an ISO 7 clean room (circles), and a set obtained by hand in ambient air (triangles).

lifetime obtained with the van der Waals instability. As observed, increasing the critical rupture thickness by only few hundreds of nanometers affects significantly the lifetime of antibubbles. Moreover, to reproduce all the data (circles) obtained in a clean environment with our antibubble generator, one has to consider a critical thickness of $0.5 \mu \mathrm{m}$ at most. This value is consistent with the fact that experiments where conducted in an ISO 7 cleanroom where only particles of diameter larger than $0.3 \mu \mathrm{m}$ are filtered. Assuming intermolecular forces are finally responsible for the rupture, a particle of $0.3 \mu \mathrm{m}$ might reasonably destabilize a shell of $0.5 \mu \mathrm{m}$ thickness.

With the aim to confirm this rupture mechanism, we compare in Fig. 12 the lifetime of antibubbles produced in a clean environment to antibubbles produced in ambient air by hand, following the same procedure than in [18]. Without excluding a possible contribution of gas dissolution (see previous section), it is shown that antibubbles produced in ambient air have a smaller 
Table 4: Occurrence ratio of antibubbles with entrapped dust particles, depending on the antibubble radius $R$, particle size and environment. The volume of entrapped air is calculated as $4 \pi R^{2} h_{0}$, with $h_{0}=4 \mu \mathrm{m}$. The data for ambient air are mean values taken from [55].

\begin{tabular}{|c|c|c|c|c|}
\cline { 2 - 5 } \multicolumn{1}{c|}{} & \multicolumn{2}{c|}{ Iso 7 clean room } & \multicolumn{2}{c|}{ Ambient air } \\
\cline { 2 - 5 } \multicolumn{1}{c|}{} & $\geq 0.5 \mu \mathrm{m}$ & $\geq 0.3 \mu \mathrm{m}$ & $>0.5 \mu \mathrm{m}$ & $\geq 0.3 \mu \mathrm{m}$ \\
\hline Nbr of particles $/ \mathrm{m}^{3}$ & 352000 & N.A. & $\sim 5 \times 10^{7}$ & $\sim 2.5 \times 10^{8}$ \\
\hline \hline$R=2.5 \mathrm{~mm}$ & $1: 9000$ & & $1: 64$ & $1: 13$ \\
\hline$R=10 \mathrm{~mm}$ & $1: 565$ & & $1: 4$ & $1: 1$ \\
\hline
\end{tabular}

lifetime than those produced in a clean environment. These results confirm the strong influence of dust particles on antibubble lifetime, as they are more numerous and larger in ambient air than in a clean room. Actually, Table 4 shows the occurrence ratio for an antibubble to contain entrapped dust particles in the air shell for both environments.

Note on the one hand that the number of particles of size lower than $0.5 \mu \mathrm{m}$ in an ISO 7 clean room is not available but the gas used with our antibubble generator came from a filtered air compressor. On the other hand, the occurrence ratio reported in Table 4 for airborne particles is only a rough estimate as it corresponds to outdoor measurements made by [55], assuming that a correlation exists with indoor airborne particle concentration, as reported in [56]. Nevertheless, the occurrence ratio of dust-loaded antibubble is extremely low in a clean room, while it is much higher in ambient air, and especially for larger antibubbles, for which every antibubble of $10 \mathrm{~mm}$ radius should contain at least one particle of $0.3 \mu \mathrm{m}$. These data perfectly corroborate the observations reported in Fig. 12 for antibubble lifetimes.

With the aim to confirm the influence of dust particles as the main cause for the antibubble collapse, we can consider the cumulative distribution function $(\mathrm{CDF})$ of the lifetimes, which can naturally be described by the twoparameter Weibull distribution of the form

$$
\mathrm{CDF}_{\mathrm{W}}=1-\exp ^{-\left(\frac{t_{\text {life }}}{t_{0}}\right)^{k}}
$$

where $k$ and $t_{0}$ are the shape and the scale parameters, respectively. In previous works $[18,25,31]$, the distribution of the antibubble lifetimes was found to follow a Markovian distribution, i.e. with $k=1$. This means that 


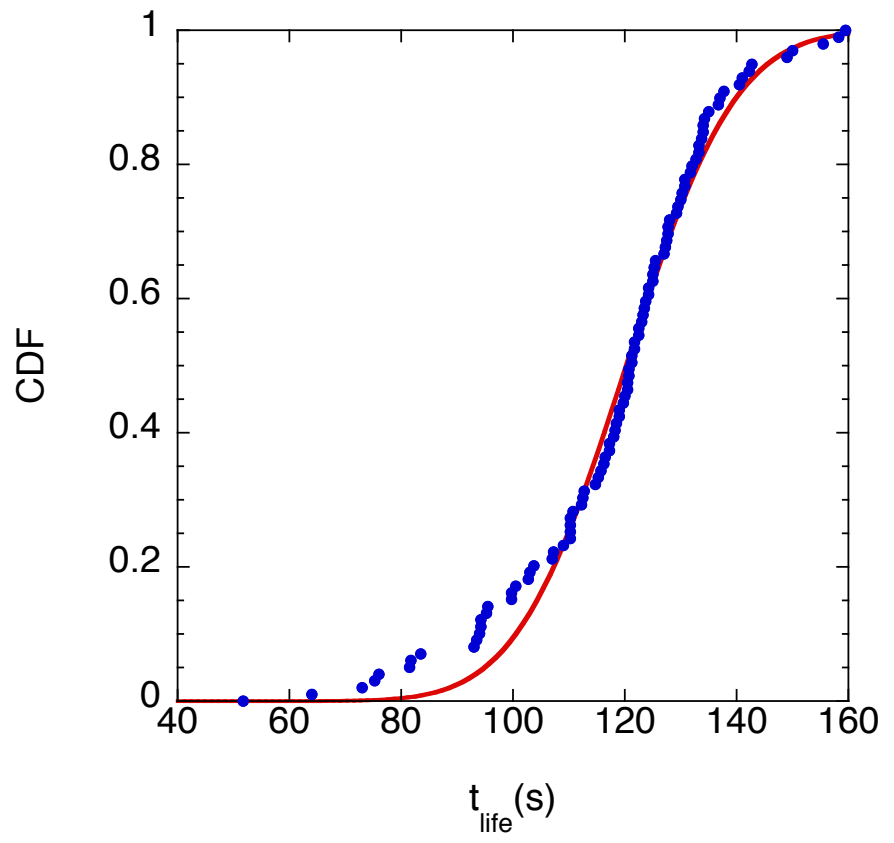

Figure 13: Cumulative distribution function of the lifetimes of antibubbles having a radius between 1.9 and $2.1 \mathrm{~mm}$ (data extracted from Fig. 9). The red curve corresponds to a fit using the $\mathrm{CDF}_{\mathrm{G}}$ of the Gaussian distribution with the mean $\bar{t}_{\text {life }}=120 \mathrm{~s}$ and the variance $\sigma=15.5$.

the collapse probability remained constant during the whole lifetime of the antibubble. The antibubbles were made by hand without any control on the radius, the amount of dust particles and the gas content in the liquid phase. In Fig. 13, we report the CDF of the lifetimes for the antibubbles produced in controlled conditions using our antibubble generator and select the antibubbles having a radius between 1.9 and $2.1 \mathrm{~mm}$ only, as extracted from the data of Fig. 9. The red curve corresponds to the CDF of a Gaussian, namely

$$
\mathrm{CDF}_{\mathrm{G}}=\frac{1}{2}\left[1+\operatorname{erf}\left(\frac{t_{\text {life }}-\bar{t}_{\text {life }}}{\sigma \sqrt{2}}\right)\right],
$$

where $\bar{t}_{\text {life }}$ is the average lifetime and $\sigma$ the standard deviation. Remarkably, the best fit with a Gaussian distribution indicates that the antibubble collapse results from a deterministic process rather than a stochastic process, as 
it should inherently be the case for coalescence when dominated by thermal fluctuations [57]. This was confirmed by fitting the data using $\mathrm{CDF}_{\mathrm{W}}$ (not shown) that gave a shape factor $k=9.1$ and a scale factor $t_{0}=126 \mathrm{~s}$. Such a high shape factor in particular suggested a better fitting by a normal distribution. Consequently, the deterministic character of the film collapse that we have found confirm the dominant role of dust particles in determining the antibubble lifetime. And this was included in the modeling by imposing a critical thickness for rupture associated to the maximum size of the filtered particles.

\subsection{Other surfactants}

In order to complete the study, we have performed additional experiments with another surfactant, namely $\mathrm{C}_{12} \mathrm{E}_{6}$. Like TX-100, it is a non-ionic surfactant with a long adsorption timescale of about $100 \mathrm{~s}$ [29, 43], i.e. comparable to the lifetime of antibubbles. This feature is crucial for the Marangoni effect to be important and for the validity of the model that relies on the insoluble limit [35].

For this mixture, we have improved the precision on the bulk saturation to $\beta=0.99 \pm 0.01$ by adding a temperature control, and as for the measurements of the initial thickness $h_{0}$, two sizes of injector have been used to widen the range of antibubble radii to approximately one decade, i.e. from 0.5 to 5 $\mathrm{mm}$. The surface elasticity value is left to $E_{s}=0.2 \mathrm{mN} / \mathrm{m}$ [35], since the results are not sensitive to variation of $E_{s}$ above the "rigidity" threshold, i.e. for $M a \gg 0.03$, which is also guaranteed here.

Results are plotted in Fig. 14. As predicted by our theory, antibubbles with $R=5 \mathrm{~mm}$ can live about 10 times longer than in ambiant air, i.e. about ten minutes instead of one, when made in a clean room with our antibubble generator and a liquid almost saturated in gas. As for TX-100, the smaller lifetime for antibubbles made by hand is probably due to both larger particles in the atmosphere and uncontrolled saturation of the liquid with gas.

These results with another surfactant confirms that controlling the liquid saturation is crucial to allow quantitative comparison between experiment and theory. And that the dispersion of data is most probably due to the presence of dust particles in the atmosphere.

\subsection{Three-dimensional effects}

A last source of the lifetime dispersion that we can think of, is the threedimensional effect that can lead to azimuthal destabilisation of the air shell. 


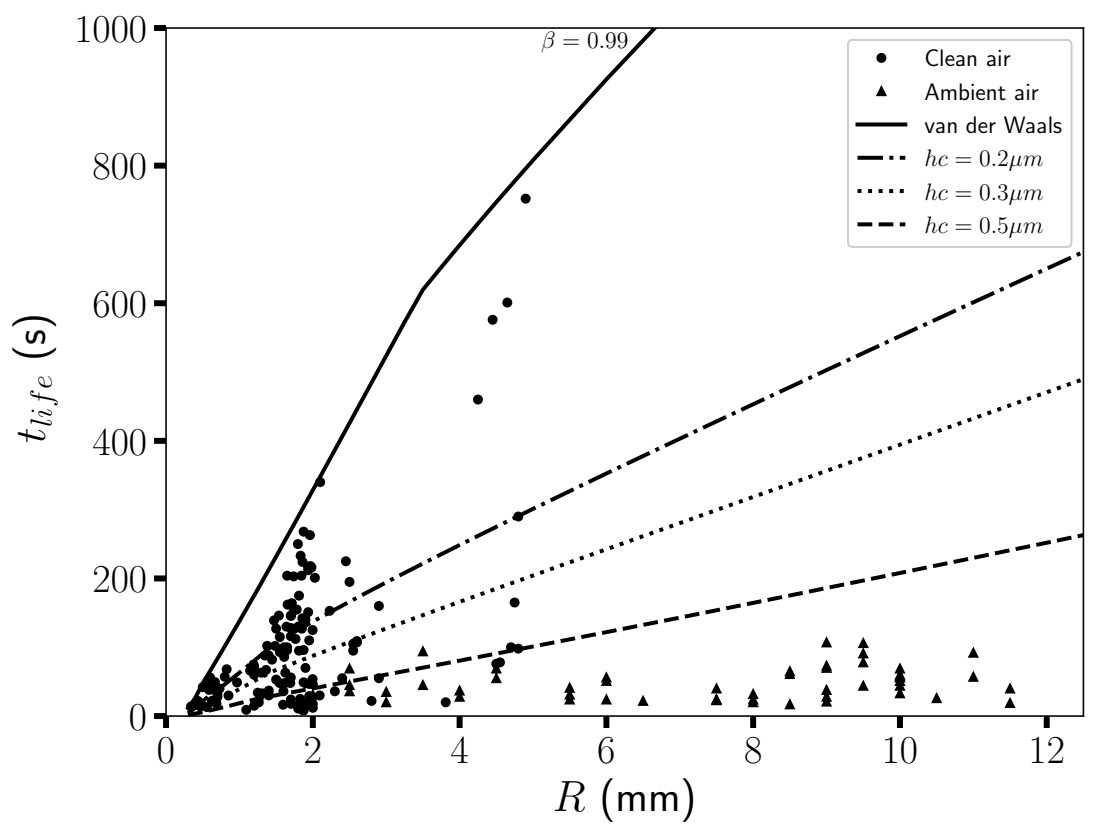

Figure 14: Same as for Fig. 12 with $\mathrm{C}_{12} \mathrm{E}_{6}$ at $10 \mathrm{CMC}$ and $\beta=0.99$.

Some channeling has actually been reported in antibubbles [18, 19], the mechanism of which has not been identified yet, even though the large observed wavelength suggests that it takes its origin in a hydrodynamic instability rather than in van der Waals interactions. One can be tempted to state that such channeling would shorten the lifetime in a significant manner, as most of the gas drainage would take place in the thicker channels, which are less hydrodynamically resistive than the thin shell elsewhere. Nevertheless, and as it can be observed in a video [58], the lifetime can instead be increased by three-dimensional instabilities reminiscent of the marginal regeneration encountered in soap films [59]. Indeed, as rationalized by Bruinsma [60] for soap films, patches of thicker air film could rise and induce gravity-driven convection into the air shell, sustaining the antibubble for a longer time.

In order to evaluate if these three-dimensional effects were present in our experiments, we have visualized antibubbles using monochromatic light. Figure 15 shows three pictures of antibubbles taken from the side (a) and from the bottom (b-c). Black fringes can be seen and arise from interferences between the light being reflected on both interfaces of the air shell. All 

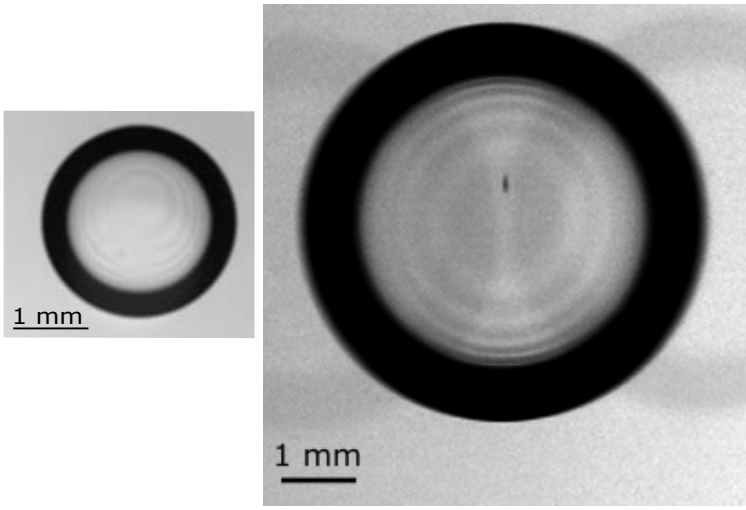

(a)

(b)

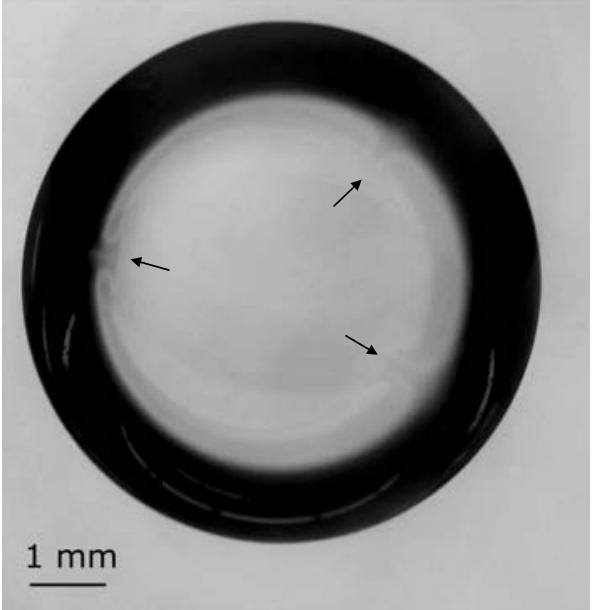

(c)

Figure 15: Pictures of antibubbles illuminated by a monochromatic light: (a) antibubble made by our generator in the clean room using TX-100 and observed from the side; it shows regular interference fringes indicating a uniform drainage, (b) antibubble made by hand in ambient air using TX-100 and observed from the bottom; it shows concentric interference fringes, (c) same as (b) but using commercial detergent (Dreft ${ }^{\complement}$ ); it shows non-regular interferences fringes, as pointed out by the arrows, indicating a non-uniform drainage. Each picture have the same scale. 
antibubbles obtained with TX-100 that we have checked showed uniform fringes as in Fig.15a-b, which suggests that no 3D effect can be responsible for the premature deaths of antibubbles observed in our experiments. Actually, we were not able to observe 3D effects, unless using commercial detergent, as shown in Fig. $15 \mathrm{c}$ with Dreft ${ }^{\circledR}$. In fact, the only picture in literature showing 3D effects can be found in [18] (Fig. 3), for which commercial detergent (Palmolive $\left.{ }^{(}\right)$was also used, even though not explicitly mentioned in that reference. Worth to mention is that the 3D effects observed in Fig. 15c were observed after some delay of about $100 \mathrm{~s}$ and did not immediately lead to rupture, suggesting as mentioned above a stabilizing mechanism similar to the marginal regeneration. Provided the lifetimes of hand-made antibubbles plotted in Figs. 12 and 14 (triangles) were all shorter than 100s, one can state with confidence that the dispersion observed on the lifetimes are effectively due to dust particles, as explained in section 4.4, rather than to $3 \mathrm{D}$ effects.

Note finally that our theoretical model relies on the axisymmetry of the antibubble and that the good agreement with experimental data, at least for the longest living antibubble, supports the argument that antibubbles generated in this work were indeed axisymmetric, as illustrated by the concentric interference fringes in Fig.15b.

\section{Conclusions}

This work reports an extended study on antibubbles generated and stabilized with nonionic and small surfactant molecules that have a sufficiently low surface adsorption time for the Marangoni stress to be present. By sequentially investigating with our on-demand antibubble generator all effects capable of influencing the antibubble lifetime, we have de-correlated each contribution, leading to the following findings:

(i) The lifetime of an antibubble is not controlled by surface viscosity for small surfactant molecules, but using an inviscid interface in the modelling did not yield representative results;

(ii) Surface elasticity represents the main mechanism to increase the lifetime of antibubbles, provided the Marangoni number, measuring the surface rigidity, is larger than a threshold value $M a_{\mathrm{th}} \approx 0.03$;

(iii) Above this threshold, the lifetime is shown to be almost independent on the surface elasticity, hence on the bulk concentration of the surfactant mixture; 
(iv) Avoiding interference from gas dissolution and dust particles, antibubble lifetime is proven to increase with the antibubble radius;

(v) Initial shell thickness of the antibubble (of about $4 \mu \mathrm{m}$ ) has no crucial influence on the lifetime and does actually not depend significantly on the antibubble radius;

(vi) Accounting for gas dissolution in the liquid, even if it is close to saturation, is crucial to obtain qualitative agreement with modeling;

(vii) Even though the deterministic parts of coalescence is often difficult to identify, as pointed out in the recent review by Kamp et al. [61], our careful experiments allowed with confidence to attribute it to the presence of dust particles whose maximum size was bounded by filtration in both the clean room and the injected antibubble gas phase. On the contrary unfiltered dust particles that are also more numerous in the atmosphere provoke a higher occurrence of premature antibubble collapses, conferring the stochastic character of the antibubble lifetime distributions observed previously in literature;

(viii) The occurrence ratio of dust-induced antibubble collapse is higher for large antibubbles than for small ones, as the volume of embedded gas scales with the square of the antibubble radius.

(ix) Three-dimensional flow effects were never observed in antibubbles made with TX-100, in the case of which comparisons with axisymmetric modeling are valid.

(x) As long as 3D effects are concerned, there is a fundamental difference between pure surfactant mixtures used in this work and commercial detergents, which deserves further investigations.

Based on these findings obtained with Triton-X-100 mixtures, we have performed lifetime measurements with $\mathrm{C}_{12} \mathrm{E}_{6}$ in highly saturated liquids, and have shown that the lifetime of antibubbles can be increased by a factor ten, if obtained in a clean room environment, as compared to those obtained in ambient air. It is thus demonstrated through the present study that optimizing the lifetime of antibubbles is feasible, paving the way towards future applications requiring controlling the lifetime, like for instance in vectorization or all-aqueous emulsification. 


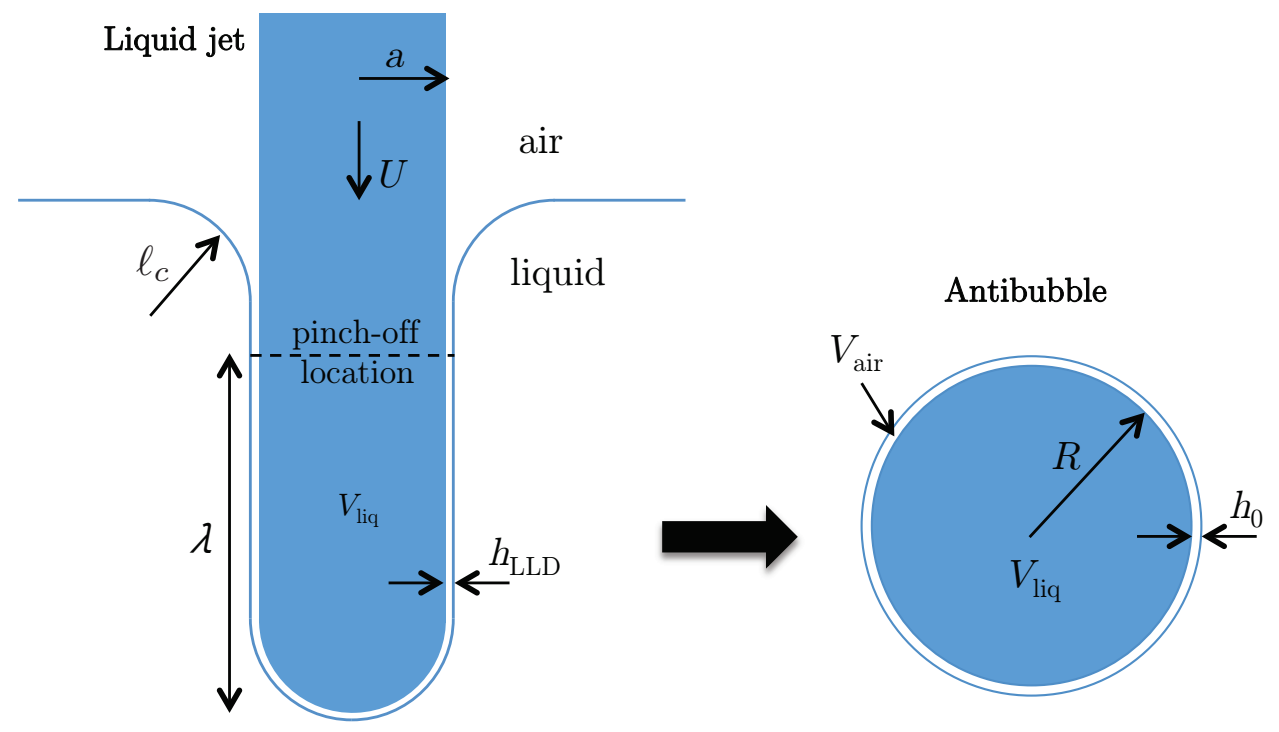

Figure A.16: Sketch of the antibubble formation.

\section{Acknowledgements}

We thank Joe Samaniuk for his assistance in measuring surface elasticities. We are also grateful to Nikolai Denkov and Dominique Langevin for fruitful discussions about three-dimensional drainage. We thank the IAP 7/38 MicroMAST project supported by BELSPO for financial support. S.D. and B.S. thank the F.R.S-FNRS for the financial support.

\section{Appendix A.}

\section{Origin of the initial shell thickness in an antibubble}

This appendix aims at estimating the initial film thickness of the antibubble's air shell and capture a possible dependence with the antibubble radius. As shown in Fig. 7, the antibubble formation consists in a jet that penetrates into a liquid bath, entraining an annular film of air and pinching-off by the Rayleigh-Plateau instability [10]. This sequence is sketched in Fig. A.16, along with the relevant parameters. Note that the gas is considered to be air in this example. Following [62], who assumed the jet to behave like a solid cylinder moving at constant speed $U$, the thickness of the entrained air film can be evaluated by the Landau-Levich-Derjaguin law, namely

$$
h_{\mathrm{LLD}}=\frac{1.34}{C} 4^{2 / 3} C a^{2 / 3}
$$


where $C$ is a curvature factor driving the capillary suction in the air film, and $C a=\mu_{g} U / \gamma_{0}$ is the capillary number. We used the factor $4^{2 / 3}$ instead of $2^{2 / 3}$ by [62] to account for the surfactant-induced rigidity of the outer interface, contributing to the maximum thickening of the air film [35]. In the case of the jet radius $a$ much larger than the capillary length $\ell_{c}=\sqrt{\gamma /\left(\rho_{\ell} g\right)}$, namely $a \gg \ell_{c}$, the capillary pressure driving the suction at the static meniscus is $\gamma_{0} \sqrt{2} / \ell_{c}$, like for flat films in perfectly wetting conditions [63]. In the opposite case, namely $a \ll \ell_{c}$, the driving capillary pressure is essentially $\gamma / a$ [64]. Therefore, in the intermediate case for which $a \sim \ell_{c}$, the driving capillary pressure is the addition of the two contributions, which thus lead to the following curvature factor

$$
C=\left(\frac{\sqrt{2}}{\ell_{c}}+\frac{1}{a}\right) .
$$

Based on linear stability theory, let us parametrise the length of the jet segment that will destabilize to form an antibubble by $\lambda=\alpha a$, where $\alpha$ characterizes the way the Rayleigh-Plateau instability is triggered. For a jet destabilising into the air, the most amplified mode corresponds to $\alpha=9.01$ [65], but the cut-off wavelength is for $\alpha=6.28$. Provided the jet instability is strongly influenced by the presence of the outer liquid, inducing in turn finite amplitude perturbations at the impact, we will consider in the following that $\alpha$ can take any value in the interval $[6.28,9.01]$.

Matching the liquid volume $V_{\text {liq }}$ of the destabilised jet segment, $\pi a^{2} \lambda$, with the liquid volume embedded in the antibubble, $4 \pi R^{3} / 3$, provided $h_{0} / R \ll 1$, yields

$$
R=\sqrt[3]{\frac{3 \alpha}{4}} a
$$

which shows that the antibubble size is essentially proportional to the jet radius. Matching now the volume $V_{\text {air }}$ of the entrained air film, $2 \pi a \lambda h_{\mathrm{LLD}}$, with the air volume of the antibubble, $4 \pi R^{2} h_{0}$, and using (A.3), yield

$$
h_{0}=\sqrt[3]{\frac{2 \alpha}{9}} h_{\mathrm{LLD}}
$$

Incorporating (A.1) and (A.2) into (A.4), while eliminating a using (A.3) finally gives a relationship between the initial film thickness and the antibubble radius. Taking $U=0.1 \mathrm{~m} / \mathrm{s}$ and $\alpha=6.28$ give the solid curve plotted in Fig. 10, using for the other parameters their values from Table 1. 


\section{Bibliography}

[1] W. Hughes, A. R. Hughes, Liquid drops on the same liquid surface, Nature 129 (1932) 59.

[2] C. L. Stong, The amateur scientist, Scientific American: Curious bubbles in which a gas encloses a liquid instead of the other way around 230 (4) (1974) 116-122.

[3] Y. Iida, T. Takashima, Direct-contact heat transfer characteristics: Evaporation of a drop dropped onto a hot liquid surface, International Journal of Heat and Mass Transfer 23 (9) (1980) 1263 - 1271.

[4] T. Nosoko, Y. Mori, Vaporization of drops of a denser, volatile liquid dropped onto a surface of another liquid, Transaction of the ASME 107 (1985) 384-391.

[5] K. Johansen, S. Kotopoulis, M. Postema, Ultrasonically driven antibubbles encapsulated by newtonian fluids for active leakage detection, in: Proceedings of the International MultiConference of Engineers and Computer Scientists, Vol II, 2015, pp. 1-5.

[6] S. Kotopoulis, K. Johansen, O. Gilja, A. Poortinga, M. Postema, Acoustically active antibubbles, Acta Physica Polonica A 127 (1) (2015) 99102.

[7] M. Postema, A. Novell, C. Sennoga, A. T. Poortinga, A. Bouakaz, Harmonic response from microscopic antibubbles, Applied Acoustics 137 (2018) $148-150$.

[8] J. E. Silpe, D. W. McGrail, Magnetic antibubbles: Formation and control of magnetic macroemulsions for fluid transport applications, Journal of Applied Physics 113 (17) (2013) 17B304.

[9] S. Dorbolo, H. Caps, N. Vandewalle, Fluid instabilities in the birth and death of antibubbles, New Journal of Physics 5 (1) (2003) 161.

[10] P. G. Kim, H. A. Stone, Dynamics of the formation of antibubbles, Europhys. Lett. 83 (2008) 54001.

[11] A. Tufaile, J. C. Sartorelli, Bubble and spherical air shell formation dynamics, Phys. Rev. E 66 (2002) 056204. 
[12] M. Postema, F. ten Cate, G. Schmitz, N. de Jong, A. van Wamel, Generation of a droplet inside a microbubble with the aid of an ultrasound contrast agent: First result, Letters in Drug Design \& Discovery 4 (1) (2007) 74-77.

[13] A. T. Poortinga, Micron-sized antibubbles with tunable stability, Colloids and Surfaces A: Physicochemical and Engineering Aspects 419 (2013) 15-20.

[14] J. E. Silpe, J. K. Nunes, A. T. Poortinga, H. A. Stone, Generation of antibubbles from core-shell double emulsion templates produced by microfluidics, Langmuir 16.

[15] Y. Shen, L. Hu, W. Chen, H. Xie, X. Fu, Drop encapsulated in bubble: A new encapsulation structure, Phys. Rev. Lett. 120 (2018) 054503.

[16] L. Bai, W. Xu, P. Wu, W. Lin, C. Li, D. Xu, Formation of antibubbles and multilayer antibubbles, Colloids and Surfaces A: Physicochemical and Engineering Aspects 509 (2016) 334 - 340.

[17] D. Beilharz, A. Guyon, E. Li, M. Thoraval, S. Thoroddsen, Antibubbles and fine cylindrical sheets of air, J. Fluid Mech. 779 (2015) 87.

[18] S. Dorbolo, E. Reyssat, N. Vandewalle, D. Quéré, Aging of an antibubble, EPL (Europhysics Letters) 69 (6) (2005) 966.

[19] W. Suhr, Gaining insight into antibubbles via frustrated total internal reflection, European Journal of Physics 33 (2) (2012) 443.

[20] A. T. Poortinga, Long-lived antibubbles: Stable antibubbles through Pickering stabilization, Langmuir 27 (6) (2011) 2138-2141.

[21] P. G. Kim, J. Vogel, Antibubbles: factor that affects their stability, Coll. Surf. A 289 (2006) 237-244.

[22] J. Zou, W. Wang, C. Ji, Bouncing antibubbles, Experiments in Fluids 57 (9) (2016) 147.

[23] J. Zou, C. Ji, B. Yuan, X. Ruan, X. Fu, Collapse of an antibubble, Physical Review E 87 (6) (2013) 061002. 
[24] D. N. Sob'yanin, Theory of the antibubble collapse, Physical Review Letters 114 (10).

[25] S. Dorbolo, D. Terwagne, R. Delhalle, J. Dujardin, N. Huet, N. Vandewalle, N. Denkov, Antibubble lifetime: Influence of the bulk viscosity and of the surface modulus of the mixture, Colloids and Surfaces A: Physicochemical and Engineering Aspects 365 (2010) 43-45.

[26] K. Golemanov, N. D. Denkov, S. Tcholakova, M. Vethamuthu, A. Lips, Surfactant mixtures for control of bubble surface mobility in foam studies, Langmuir 24 (2008) 9956.

[27] B. Scheid, S. Dorbolo, L. R. Arriaga, E. Rio, Antibubble dynamics: The drainage of an air film with viscous interfaces, Phys. Rev. Lett. 109 (2012) 264502 .

[28] J. Emile, A. Salonen, B. Dollet, A. Saint-Jalmes, A systematic and quantitative study of the link between foam slipping and interfacial viscoelasticity, Langmuir 25 (2009) 13412-13418.

[29] S.-Y. Lin, Y.-C. Lee, M.-J. Shao, Adsorption kinetics of $c_{1} 2 \mathrm{e}_{6}$ at the air-water interface, J. Chin. Inst. Chem. Engrs. 33 (2002) 631.

[30] Z. A. Zell, A. Nowbahar, M. Mansard, L. G. Leal, S. S. Deshmukh, J. M. Mecca, C. J. Tucker, T. M. Squires, Surface shear inviscidity of soluble surfactants, Proc. Nat. Am. Soc. 111 (10) (2014) 3677-3682.

[31] B. Scheid, J. Zawala, S. Dorbolo, Gas dissolution in antibubble dynamics, Soft Matter 10 (2014) 7096-7102.

[32] Anti-bubble generator (Jun. 2017).

URL https://data.epo.org/publication-server/rest/v1.0/ publication-dates/20170809/patents/EP3202491NWA1/document. pdf

[33] J. C. Slattery, L. Sagis, E.-S. Oh, Interfacial transport phenomena, 2nd Edition, Springer Verlag, 2007.

[34] H. A. Stone, A simple derivation of the time-dependent convectivediffusion equation for surfactant transport along a deforming interface, Phys. Fluids A 2 (1) (1990) 111-112. 
[35] L. Champougny, B. Scheid, F. Restagno, J. Vermant, E. Rio, Surfactantinduced rigidity of interfaces: a unified approach to free and dip-coated films, Soft Matter 11 (14) (2015) 2758-2770.

[36] C. F. Curtiss, J. O. Hirschfelder, Integration of stiff equations, Proceedings of the National Academy of Sciences 38 (3) (1952) 235-243.

[37] S. Vandebril, A. Franck, G. Fuller, M. P., J. Vermant, A double wall-ring geometry for interfacial shear rheometry, Rheol Acta 49 (2010) 131-144.

[38] C. J. W. Breward, P. D. Howell, The drainage of a foam lamella, Journal of Fluid Mechanics 458 (2002) 379?406.

[39] L. W. Schwartz, R. V. Roy, Modeling draining flow in mobile and immobile soap films, J. Coll. Interf. Sc. 218 (1999) 309-323.

[40] Y. Vitry, S. Dorbolo, B. Scheid, Antibubble generator (Dec 2014). URL https://youtu.be/149BoJCcf_I

[41] R. Battino, T. R. Rettich, T. Tominaga, The solubility of nitrogen and air in liquids, Journal of Physical and Chemical Reference Data 13 (2) (1984) 563.

[42] C. Stubenrauch, O. J. Rojas, J. Schlarmann, P. M. Claesson, Interactions between nonpolar surfaces coated with the nonionic surfactant hexaoxyethylene dodecyl ether C12e6 and the origin of surface charges at the air/water interface, Langmuir 20 (12) (2004) 4977-4988.

[43] V. B. Fainerman, S. V. Lylyk, E. V. Aksenenko, A. V. Makievski, J. T. Petkov, J. Yorke, R. Miller, Adsorption layer characteristics of Triton surfactants: 1. Surface tension and adsorption isotherms, Colloids and Surfaces A: Physicochemical and Engineering Aspects 334 (2009) 1-7.

[44] K.-D. Wantke, H. Fruhner, J. Fang, K. Lunkenheimer, Measurements of the surface elasticity in medium frequency range using the oscillating bubble method, Journal of Colloid and Interface Science 208 (1) (1998) $34-48$.

[45] N.-S. Cheng, Formula for the viscosity of a glycerol?water mixture, Ind. Eng. Chem. Res. 47 (2008) 3285-3288. 
[46] A. Volk, C. J. Kähler, Density model for aqueous glycerol solutions, Experiments in Fluids 59 (5) (2018) 75.

[47] M. S. Bhamla, C. E. Giacomin, C. Balemans, G. G. Fuller, Influence of interfacial rheology on drainage from curved surfaces, Soft Matter 10 (2014) 6917-6925.

[48] Y. Matsumoto, T. Uda, S. Takagi, The effect of surfactant on rising bubbles, in: S. Balachandar, A. e. Prosperetti (Eds.), IUTAM Symposium on Computational Approaches to Multiphase Flow. Fluid Mechanics and Its Applications, Vol. 81, Springer, Dordrecht, 2006, pp. 311-321.

[49] H. Allen, On the motion of a sphere in a viscous fluid, Philosophical Magazine 50 (1900) 323-338.

[50] R. Turton, O. Levenspiel, A short note on the drag correlation for spheres, Powder Technology 47 (1986) 83-86.

[51] P. Brown, D. F. Lawler, Sphere drag and settling velocity revisited, Journal of Environmental Engineering 129 (2003) 222-231.

[52] J. Israelachvili, Intermolecular and Surface Forces, 3rd Edition, Academic Press, Amsterdam, 2011.

[53] N. D. Denkov, K. G. Marinova, S. S. Tcholakova, Mechanistic understanding of the modes of action of foam control agents, Advances in Colloid and Interface Science 206 (2014) 57-67.

[54] A. Evangelio Sánchez, Generacion controlada de microburbujas y microemulsiones. Estabilidad de burbujas en interfases, PhD Thesis, Universidad de Sevilla, 2016.

[55] A. Tittarelli, A. Borgini, M. Bertoldi, E. De Saeger, A. Ruprecht, R. Stefanoni, G. Tagliabue, P. Contiero, P. Crosignani, Estimation of particle mass concentration in ambient air using a particle counter, Atmospheric Environment 42 (2008) 8543?8548.

[56] L. Morawska, C. He, J. Hitchins, D. Gilbert, S. Parappukkaran, The relationship between indoor and outdoor airborne particles in the residential environment, Atmospheric Environment 35 (2001) 3463-3473. 
[57] G. Charles, S. Mason, The coalescence of liquid drops with flat liquid/liquid interfaces, Journal of Colloid Science 15 (3) (1960) 236 - 267.

[58] S. Dorbolo, Antibubble observed with monochromatic light (bottom view) (Nov 2009).

URL https://youtu.be/JtrTpEmJZsA

[59] K. J. Mysels, K. Shinoda, S. Frankel, Soap Films: Studies of their Thinning and a Bibliography, Pergamon, New York, 1959.

[60] R. Bruinsma, Theory of hydrodynamic convection in soap films, Physica A: Statistical Mechanics and its Applications 216 (1) (1995) 59 - 76.

[61] J. Kamp, J. Villwock, M. Kraume, Drop coalescence in technical liquid/liquid applications: a review on experimental techniques and modeling approaches, Rev. Chem. Eng. 33 (1) (2017) 1-47.

[62] E. Lorenceau, D. Quéré, J. Eggers, Air entrainment by a viscous jet plunging into a bath, Physical Review Letters 93 (25) (2004) 254501.

[63] P.-G. de Gennes, F. Brochart-Wyart, D. Quéré, Gouttes, Bulles, Perles et Ondes, Belin (Paris), 2005.

[64] D. Qur, Fluid coating on a fiber, Annual Review of Fluid Mechanics 31 (1) (1999) 347-384.

[65] F. Charru, Hydrodynamic Instabilities, Cambridge University Press, 2011. 